$\underline{\text { Preprint typeset in JHEP style - PAPER VERSION }}$

WITS-CTP-061

\title{
Surprisingly Simple Spectra
}

\author{
Vincent De Comarmond ${ }^{1}$, Robert de Mello Koch $^{1,2}$ and Katherine Jefferies ${ }^{1}$ \\ 1 National Institute for Theoretical Physics, \\ Department of Physics and Centre for Theoretical Physics, \\ University of the Witwatersrand, \\ Wits, 2050, South Africa \\ ${ }^{2}$ Stellenbosch Institute for Advanced Studies, \\ Stellenbosch, South Africa \\ E-mail: Vincent.Decomarmond@students.wits.ac.za, \\ robert@neo.phys.wits.ac.za, Katherine.Jefferies@students.wits.ac.za
}

\begin{abstract}
The large $N$ limit of the anomalous dimensions of operators in $\mathcal{N}=4$ super Yang-Mills theory described by restricted Schur polynomials, are studied. We focus on operators labeled by Young diagrams that have two columns (both long) so that the classical dimension of these operators is $O(N)$. At large $N$ these two column operators mix with each other but are decoupled from operators with $n \neq 2$ columns. The planar approximation does not capture the large $N$ dynamics. For operators built with 2, 3 or 4 impurities the dilatation operator is explicitly evaluated. In all three cases, in a certain limit, the dilatation operator is a lattice version of a second derivative, with the lattice emerging from the Young diagram itself. The one loop dilatation operator is diagonalized numerically. All eigenvalues are an integer multiple of $8 g_{Y M}^{2}$ and there are interesting degeneracies in the spectrum. The spectrum we obtain for the one loop anomalous dimension operator is reproduced by a collection of harmonic oscillators. This equivalence to harmonic oscillators generalizes giant graviton results known for the BPS sector and further implies that the Hamiltonian defined by the one loop large $N$ dilatation operator is integrable. This is an example of an integrable dilatation operator, obtained by summing both planar and non-planar diagrams.
\end{abstract}

KEYwords: Giant Gravitons, AdS/CFT correspondence, super Yang-Mills theory. 


\section{Contents}

1. Introduction 1

2. Action of the Dilatation Operator 4

$\begin{array}{ll}\text { 3. Excited Giant Graviton Bound States } & 7\end{array}$

4. Simple Projectors $\quad 8$

5. The Radial Direction $\quad 10$

5.1 Three Impurities $\quad 10$

5.2 Four Impurities 12

6. Numerical Results $\quad 15$

6.1 Two Impurities 15

6.2 Three Impurities $\quad 15$

6.3 Four Impurities $\quad 15$

$\begin{array}{ll}\text { 7. Discussion } & 16\end{array}$

A. Dilatation Operator for Three or Four impurities $\quad 20$

A.1 Three Impurities $\quad 20$

A.2 Four Impurities 24

B. A Discussion on Intertwiners 30

\section{Introduction}

In the last few years interesting new progress has been made in the study of the dynamics of multimatrix models. Starting from the remarkable observation[1] that the Schur polynomials are a complete basis of gauge invariant operators which diagonalize the two point function of the free $\left(g_{Y M}^{2}=0\right)$ super Yang-Mills theory, similar bases have been found for multimatrix models[2, 3, 4, 5, 6, 7, 8, 9, 10, 11, 12]. For these bases, the two point function is diagonal and known exactly as a function of $N$ (but of 
course, at $\left.g_{Y M}^{2}=0\right)$. The fact that the $N$ dependence is known exactly suggests that these results will be useful for going beyond the planar approximation.

When would this be needed? Often "the planar limit" and "the large $N$ limit" are taken as synonyms. This is not, in general, accurate. For example, if we imagine computing the two point correlator of an operator with a bare dimension $\Delta$ of most $\Delta \sim J$ with $\frac{J^{2}}{N} \ll 1$, then summing the planar diagrams will capture the large $N$ limit. For operators with a dimension larger than this, exploding combinatoric factors overpower the nonplanar $\left(\frac{1}{N^{2}}\right)$ suppression and the planar approximation is completely ineffective[13]. In this scenario, to get the correct large $N$ limit, it is necessary to sum a lot more than just the planar diagrams. On general grounds we expect the large $N$ limit to be simpler than the full theory[14]. The planar diagrams are a small subset of all possible diagrams, so that it is quite natural to expect that summing only the planar diagrams will give a much simpler problem. Why should the large $N$ limit be simple when one needs to sum much more than just the planar diagrams? The answer to this question will probably not be general, but rather will depend on the specific dynamical problem considered and must be answered case by case. A very pedestrian approach is simply to compute the large $N$ limit and then to look for simplifications. This has been accomplished $[15,16,17]$ in a number of interesting examples including LLM geometries $[18,19]$ and the near horizon geometry of a bound state of giant gravitons[20]. The results are remarkably simple. Indeed, as an example, for $\frac{1}{2}$ BPScorrelators in the presence of $M$ giant gravitons with $M$ of order $N$, [15, 16, 17] showed that the usual $\frac{1}{N}$ expansion is replaced by a $\frac{1}{M+N}$ expansion. Further, if one expands the exact correlators (which because they are $\frac{1}{2}$ BPS do not depend on $g_{Y M}^{2}$ but only on $N$ or $N+M)$ the expansion coefficients for correlators in the background of $M$ giants are exactly the same as the expansion coefficients for correlators with no giants present! This remarkably simple result was confirmed holographically[21] by matching to graviton dynamics in the LLM geometries using the formalism of [22]. For near-BPS operators corresponding to BMN loops[23] it was argued in [24, 25, 26] that the usual 't Hooft coupling $g_{Y M}^{2} N$ is replaced by the effective 't Hooft coupling $g_{Y M}^{2}(N+M)$. For additional interesting related studies see[20, 27].

In this article we will consider the problem of computing the anomalous dimension of an operator with a bare dimension of order $N$. To answer this question, we need to go well beyond the planar limit; we find the methods and approach of $[2,3,4,5,6,7$, $8,9,10,11]$ once again surprisingly effective. The results again exhibit a remarkable simplicity - the spectrum of anomalous dimension can be matched to the spectrum of a set of oscillators! We see once again that the large $N$ limit is indeed a simple limit.

The operators we consider, restricted Schur polynomials, will be built using $O(N)$ $Z$ s and 3 or 4 "impurities" $(Y \mathrm{~s})$, where $Z$ and $Y$ are complex adjoint scalars of $\mathcal{N}=4$ 
super Yang Mills theory. The case of operators with two impurities was studied in[30]. The dilatation operator when acting on a restricted Schur polynomial, produces terms that have a combination $Z Y-Y Z$ appearing. In [30], the techniques of [4, 6] were used to separate the $Z$ and the $Y$ and then write the results as a linear combination of restricted Schur polynomials. This method is very cumbersome as it involves the inversion of a matrix. This must be done analytically so its tedious (for the case of two impurities one must invert a $6 \times 6$ matrix). More than two impurities was effectively out of reach. In this article we develop a new formula (in section 2) which avoids this matrix inversion. This allows us to handle the cases of three and four impurities without much trouble. The resulting formulas for the dilatation operator are quite lengthy (see Appendix A), but their spectrum is surprisingly simple.

Our results suggest that for the class of operators considered, the Hamiltonian defined by the dilatation operator is integrable - it is just a set of oscillators. This is an example of an integrable dilatation operator, obtained by summing both planar and non-planar diagrams.

The operators we consider can be mapped to giant gravitons[28] in spacetime[13, 1, 29]. There is in fact already a known connection between the geometry of giant gravitons and harmonic oscillators[31, 32, 33, 34, 35, 36]. Our work differs from these results in at least two important ways. Firstly, we claim that the complete spectrum (not just the BPS spectrum!) has a connection to harmonic oscillators. Secondly, we have very good control over the set of operators we consider. Our operators are dual to a two giant system. Previous studies captured the full set of BPS states and consequently were not able to distinguish (for example) giant graviton plus graviton from excited giant graviton. Our study captures only states of the two giant system. Thus, it is rather natural to associate our oscillators with excitations modes of a giant graviton. In the same way that there are oscillators in the worldsheet theory of a string describing the oscillation modes of a string, we are describing the oscillators that would be present in a world volume description of giant gravitons describing the oscillations modes of a giant graviton D3 brane.

The problem of computing anomalous dimensions for operators with a large $\mathcal{R}$ charge has been considered before by a number of authors. The restricted Schur polynomials we consider in this article are built by distributing "impurities" (given by $Y \mathrm{~s}$ ), in an operator built mainly from $Z$ s. If we replace the $Y$ s by words containing $O(\sqrt{N})$ letters (which may be $Z, Y$ or other field or derivative of a field), these words are naturally identified with open strings[37, 38, 39]. In this case the dilatation operator reproduces the dynamics of open strings ending on a giant graviton[40, 3, 4, 6]. The mixing of operators is highly constrained. Indeed, in $[4,6]$ it was shown that operators which mix can differ at most by moving one box around on the Young diagram labeling 
the operator. Another interesting basis to consider is the Brauer basis[5, 11]. This basis is built using Brauer algebra projectors. The structure constants of the Brauer algebra are $N$ dependent. There is an elegant construction of a class of BPS operators [41] in which the natural $N$ dependence appearing in the definition of the operator[42] is reproduced by the Brauer algebra projectors[41]. Finally, another very natural approach to the problem, is to adopt a basis that has sharp quantum numbers for the global symmetries of the theory $[7,9]$. The action of the anomalous dimension operator in this sharp quantum number basis is very similar to the action in the restricted Schur basis: again operators which mix can differ at most by moving one box around on the Young diagram labeling the operator[43]. For further related interesting work see $[44,45]$. Finally, for a rather general approach which correctly counts and constructs the weak coupling BPS operators see[36].

We now conclude this introduction with a description of what is to follow. In section 2 we will describe the action of the dilatation operator on restricted Schur polynomials. The main result of this section is formula (2.2) which gives a very explicit description of the action of the dilatation operator. In section 3 we will describe, in broad terms, the set of operators we consider and explain how the dynamics of the large $N$ limit simplifies. In section 4 we explain how to construct the projectors needed to evaluate the action of the dilatation operator. A very detailed description of the specific operators we consider is given in section 5 ; we also describe a limit in which our system of two giants should be well described as a system of $D 3$ brane giants plus open strings. In this limit we see that the dilatation operator reduces to a lattice version of the second derivative, with the Young diagram labels of the restricted Schur polynomials defining the lattice. In section 6 we present our numerical results and draw some general conclusions from them in section 7 . The explicit result for the dilatation operator is given in Appendix A; the intertwiners which enter into the expression for the dilatation operator are described in Appendix B.

\section{Action of the Dilatation Operator}

In this section we will study the action of the one loop dilatation operator on restricted Schur polynomials built using two complex adjoint scalars. The main result of this section is the surprisingly simple result (2.2) for the action of the dilatation operator.

We will consider the action of the one loop dilatation operator in the $S U(2)$ sector[46] of $\mathcal{N}=4$ super Yang Mills theory

$$
D=-g_{\mathrm{YM}}^{2} \operatorname{Tr}[Y, Z]\left[\partial_{Y}, \partial_{Z}\right]
$$


on the restricted Schur polynomial

$$
\chi_{R,(r, s)}\left(Z^{\otimes n}, Y^{\otimes m}\right)=\frac{1}{n ! m !} \sum_{\sigma \in S_{n+m}} \operatorname{Tr}_{(r, s)}\left(\Gamma_{R}(\sigma)\right) Z_{i_{\sigma(1)}^{i_{1}}}^{i} \cdots Z_{i_{\sigma(n)}}^{i_{n}} Y_{i_{\sigma(n+1)}}^{i_{n+1}} \cdots Y_{i_{\sigma(n+m)}}^{i_{n+m}} .
$$

The labels of our restricted Schur polynomial $\chi_{(R,(r, s))}$ are (i) $R$, which is a Young diagram with $n+m$ boxes or equivalently an irreducible representation of $S_{n+m}$, (ii) $r$, which is a Young diagram with $n$ boxes or equivalently an irreducible representation of $S_{n}$ and (iii) $s$ which is a Young diagram with $m$ boxes or equivalently an irreducible representation of $S_{m}$. The notation $\operatorname{Tr}_{(r, s)}$ implies that one should only trace over the subspace carrying the irreducible representation ${ }^{1}(r, s)$ of $S_{n} \times S_{m}$ inside the carrier space for irreducible representation $R$ of $S_{n+m}$. This trace is most concretely realized by including a projector $P_{R \rightarrow(r, s)}$ (from the carrier space of $R$ to the carrier space of $(r, s))$ and tracing over all of $R$. A simple calculation yields ${ }^{2}$

$$
\begin{aligned}
& D \chi_{R,(r, s)}\left(Z^{\otimes n}, Y^{\otimes m}\right)= \frac{g_{\mathrm{YM}}^{2}}{(n-1) !(m-1) !} \sum_{\psi \in S_{n+m}} \operatorname{Tr}_{(r, s)}\left(\Gamma_{R}((n, n+1) \psi-\psi(n, n+1))\right) \times \\
& \times Z_{i_{\psi(1)}}^{i_{1}} \cdots Z_{i_{\psi(n-1)}^{i_{n-1}}}^{i_{n}}(Y Z-Z Y)_{i_{\psi(n)}}^{i_{n}} \delta_{i_{\psi(n+1)}}^{i_{n+1}} Y_{i_{\psi(n+2)}}^{i_{n+2}} \cdots Y_{i_{\psi(n+m)}}^{i_{n+m}} .
\end{aligned}
$$

The sum over $\psi$ runs only over permutations for which $\psi(n+1)=n+1$. To perform this sum over $\psi$, write the sum over $S_{n+m}$ as a sum over cosets of the $S_{n+m-1}$ subgroup obtained by keeping precisely those permutations that satisfy $\psi(n+1)=n+1$. The result follows immediately from the reduction rule for Schur polynomials (see [47] and appendix $\mathrm{C}$ of [3])

$$
\begin{aligned}
D \chi_{R,(r, s)}= & \frac{g_{\mathrm{YM}}^{2}}{(n-1) !(m-1) !} \sum_{\psi \in S_{n+m-1}} \sum_{R^{\prime}} c_{R R^{\prime}} \operatorname{Tr}_{(r, s)}\left(\Gamma_{R}((n, n+1)) \Gamma_{R^{\prime}}(\psi)\right. \\
& \left.-\Gamma_{R^{\prime}}(\psi) \Gamma_{R}((n, n+1))\right) Z_{i_{\psi(1)}^{i_{1}}}^{i_{1}} \cdots Z_{i_{\psi(n-1)}}^{i_{n-1}}(Y Z-Z Y)_{i_{\psi(n)}}^{i_{n}} Y_{i_{\psi(n+2)}}^{i_{n+2}} \cdots Y_{i_{\psi(n+m)}}^{i_{n+m}} .
\end{aligned}
$$

The sum over $R^{\prime}$ runs over all representations that can be subduced from $R$. Concretely, $R^{\prime}$ runs over all Young diagrams that can be obtained from $R$ by dropping a single box; $c_{R R^{\prime}}$ is the weight ${ }^{3}$ of the box that must be removed from $R$ to obtain $R^{\prime}$. We will make use of the following notation for restricted characters

$$
\chi_{R,(r, s)}(\sigma)=\operatorname{Tr}_{(r, s)}\left(\Gamma_{R}(\sigma)\right)=\operatorname{Tr}\left(P_{R \rightarrow(r, s)} \Gamma_{R}(\sigma)\right) .
$$

Now, using the identity (bear in mind that $\psi(n+1)=n+1$ )

$Z_{i_{\psi(1)}}^{i_{1}} \cdots Z_{i_{\psi(n-1)}}^{i_{n-1}}(Y Z-Z Y)_{i_{\psi(n)}}^{i_{n}} Y_{i_{\psi(n+2)}}^{i_{n+2}} \cdots Y_{i_{\psi(n+m)}}^{i_{n+m}}=\operatorname{Tr}\left(((n, n+1) \psi-\psi(n, n+1)) Z^{\otimes n} Y^{\otimes m}\right)$

\footnotetext{
${ }^{1}$ In general, because $(r, s)$ can be subduced more than once, we should include a multiplicity index. We will not write this index explicitly in this article.

${ }^{2}$ Our index conventions are $(Y Z)_{k}^{i}=Y_{j}^{i} Z_{k}^{j}$.

${ }^{3}$ Recall that the weight of a box in row $i$ and column $j$ is $N-i+j$.
} 
where

$$
\operatorname{Tr}\left(\sigma Z^{\otimes n} Y^{\otimes m}\right)=Z_{i_{\sigma(1)}}^{i_{1}} \cdots Z_{i_{\sigma(n)}}^{i_{n}} Y_{i_{\sigma(n+1)}}^{i_{n+1}} \cdots Y_{i_{\sigma(n+m)}}^{i_{n+m}}
$$

and (this identity is proved in [48])

$$
\operatorname{Tr}\left(\sigma Z^{\otimes n} Y^{\otimes m}\right)=\sum_{T,(t, u)} \frac{d_{T} n ! m !}{d_{t} d_{u}(n+m) !} \chi_{T,(t, u)}\left(\sigma^{-1}\right) \chi_{T,(t, u)}(Z, Y)
$$

we obtain

$$
\begin{gathered}
D \chi_{R,(r, s)}(Z, Y)=\sum_{T,(t, u)} M_{R,(r, s) ; T,(t, u)} \chi_{T,(t, u)}(Z, Y) \\
M_{R,(r, s) ; T,(t, u)}=g_{Y M}^{2} \sum_{\substack{\psi \in S_{n+m-1} \\
\times \chi^{\prime}}} \sum_{T,(t, u)} \frac{c_{R R^{\prime}} d_{T} n m}{d_{t} d_{u}(n+m) !} \operatorname{Tr}_{(r, s)}\left(\Gamma_{R}((n, n+1)) \Gamma_{R^{\prime}}(\psi)-\Gamma_{R^{\prime}}(\psi) \Gamma_{R}((n, n+1))\right) \times \\
\end{gathered}
$$

The sum over $\psi$ can be done by using the fundamental orthogonality relation

$$
\begin{aligned}
M_{R,(r, s) ; T,(t, u)} & =-g_{Y M}^{2} \sum_{R^{\prime}} \frac{c_{R R^{\prime}} d_{T} n m}{d_{R^{\prime}} d_{t} d_{u}(n+m)} \operatorname{Tr}\left(\left[\Gamma_{R}((n, n+1)), P_{R \rightarrow(r, s)}\right] I_{R^{\prime} T^{\prime}} \times\right. \\
& \left.\times\left[\Gamma_{T}((n, n+1)), P_{T \rightarrow(t, u)}\right] I_{T^{\prime} R^{\prime}}\right) .
\end{aligned}
$$

The reader should consult Appendix B for a definition of the intertwiners $I_{R^{\prime} T^{\prime}}$. This expression for the one loop dilatation operator is exact in $N$. It is one of the key results of this article.

To obtain the spectrum of anomalous dimensions, we need to consider the action of the dilatation operator on normalized operators. The two point function for restricted Schur polynomials has been computed in [8]

$$
\left\langle\chi_{R,(r, s)}(Z, Y) \chi_{T,(t, u)}(Z, Y)^{\dagger}\right\rangle=\delta_{R,(r, s) T,(t, u)} f_{R} \frac{\operatorname{hooks}_{R}}{\operatorname{hooks}_{r} \operatorname{hooks}_{s}} .
$$

In this expression $f_{R}$ is the product of the weights in Young diagram $R$ and hooks $R$ is the product of the hook lengths of Young diagram $R$. The normalized operators can be obtained from

$$
\chi_{R,(r, s)}(Z, Y)=\sqrt{\frac{f_{R} \operatorname{hooks}_{R}}{\text { hooks }_{r} \operatorname{hooks}_{s}}} O_{R,(r, s)}(Z, Y) .
$$

In terms of these normalized operators

$$
D O_{R,(r, s)}(Z, Y)=\sum_{T,(t, u)} N_{R,(r, s) ; T,(t, u)} O_{T,(t, u)}(Z, Y)
$$




$$
\begin{aligned}
& N_{R,(r, s) ; T,(t, u)}=-g_{Y M}^{2} \sum_{R^{\prime}} \frac{c_{R R^{\prime}} d_{T} n m}{d_{R^{\prime}} d_{t} d_{u}(n+m)} \sqrt{\frac{f_{T} \operatorname{hooks}_{T} \operatorname{hooks}_{r} \operatorname{hooks}_{s}}{f_{R} \operatorname{hooks}_{R} \operatorname{hooks}_{t} \operatorname{hooks}_{u}} \times} \\
& \times \operatorname{Tr}\left(\left[\Gamma_{R}((n, n+1)), P_{R \rightarrow(r, s)}\right] I_{R^{\prime} T^{\prime}}\left[\Gamma_{T}((n, n+1)), P_{T \rightarrow(t, u)}\right] I_{T^{\prime} R^{\prime}}\right) .
\end{aligned}
$$

This last expression will be used later when we numerically study the spectrum of the dilatation operator.

\section{Excited Giant Graviton Bound States}

The goal of this section is to clearly define the class of operators being considered and to outline the approximations that can be made in the large $N$ limit.

In this article we will study restricted Schur polynomials labeled by Young diagrams with at most two columns. The number of $Z$ s appearing is $\alpha N$ where $2-\alpha \equiv \zeta \ll 1$. The number of $Y \mathrm{~s}$ appearing is fixed to be $O(1)$. These operators are dual to giant gravitons that wrap an $S^{3}$ in the $S^{5}$ of the $\operatorname{AdS}_{5} \times \mathrm{S}^{5}$ background. Since the restricted Schur polynomials furnish a suitable basis for the two giant system, we know that these operators capture all excitations (BPS and non supersymmetric) of the two giant system. For a study of excitations of the single giant system using restricted Schur polynomials see [30]. For a spacetime study of excitations of the single giant system using the Born-Infeld action see [49]. The most important result from [49, 30] for us, is that all the deformations of the single threebrane giant graviton that we consider, are supersymmetric.

The mixing of these operators with restricted Schur polynomials that have three columns (or more) is suppressed by a factor of order $\frac{1}{\sqrt{N}}$. This factor arises from the normalization of the restricted Schur polynomials: the three column restricted Schur polynomials (with one short column) have a two point function which is smaller than the two column restricted Schur polynomials by a factor of order $\frac{1}{N}[30]$. Thus, at large $N$ we can focus on the two column restricted Schur polynomials, which is a huge simplification. The analog of the statement that for operators with a dimension of $O(1)$, different trace structures do not mix is: at large $N$ restricted Schur polynomials $\chi_{R,(r, s)}$ with $R$ a Young diagram with $n$ columns, each of which has length of $O(N)$, do not mix with operators $\chi_{R^{\prime},\left(r^{\prime}, s^{\prime}\right)}$ that have $n^{\prime} \neq n$ columns. The fact that the two column restricted Schur polynomials are a decoupled sector at large $N$ is to be expected. Indeed, at large $N$ these operators correspond to a well defined stable semi-classical object in spacetime (the two giant system). We expect that $n$ column restricted Schur polynomials are also a decoupled sector at large $N$ for the same reason. 


\section{Simple Projectors}

When all Young diagrams labeling the restricted Schur polynomial have at most 2 columns, the projector $P_{R \rightarrow(r, s)}$ simplifies dramatically. The goal of this section is to explain the simplification and exploit it to efficiently build the relevant projectors.

The projector $P_{R \rightarrow(r, s)}$ projects from representation $R$ of $S_{n+m}$ to representation $(r, s)$ of $S_{n} \times S_{m}$. One issue, which complicates things considerably, is that representation $(r, s)$ can be subduced more than once when irreducible representation $R$ is decomposed into irreducible representations of the $S_{n} \times S_{m}$ subgroup. Giving a general rule to specify precisely how this multiplicity is resolved is nontrivial. For the operators we consider in this article, this problem does not arise. As soon as a system of three or more gaint gravitons are considered, it will be necessary to deal with this issue. Fortunately a well defined approach to resolving these multiplicities has been outlined in [10]. Basically, [10] considers elements in the group algebra $C S_{n+m}$ which are invariant under conjugation by $C S_{n} \times C S_{m}$. The Cartan subalgebra of these elements are the natural generalization of the Jucys-Murphy elements which define a Cartan subalgebra for $S_{n}[50]$. The multiplicities will be labelled by the eigenvalues of this Cartan subalgebra[10]. It would be very interesting to work out the details of this proposal in the context of multigiant systems.

Given $R$ we imagine removing some boxes; after removing these boxes one is left with $r$. The removed boxes are assembled to produce $s$. If we specify both $R$ and the boxes that are to be removed to obtain $r$ we obtain every representation exactly once.

For example, consider all possible operators that can be constructed from the following $R$ by removing the three boxes shown

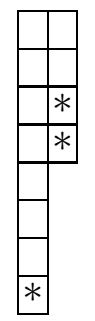

For the boxes that are removed, we must respect edges that are joined, which means the two boxes removed from the short column must remain stacked on top of each other. Thus, there are two possible irreducible representations $s$ that can be produced, implying we can subduce two possible irreducible representations of $S_{9} \times S_{3}$, by removing 
these three boxes

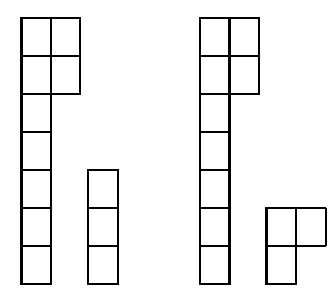

Thus, in total we'd get the following $S_{9} \times S_{3}$ irreducible representations subduced from the $R$ given above (sum over all possible ways to remove boxes to get this result)

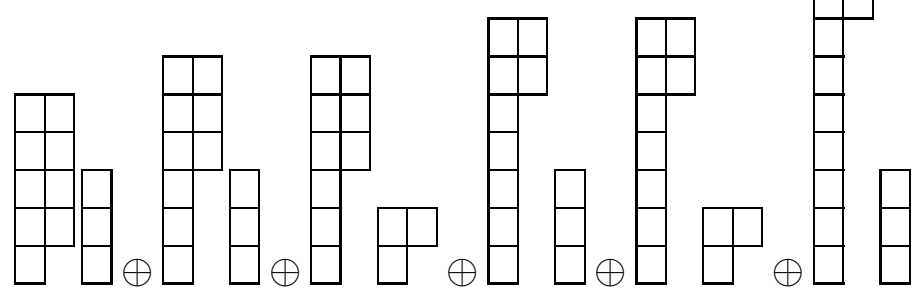

The dimensions of these representations are $42,48,96,27,54$ and 8 respectively. This sums to give 275 which is indeed the dimension of $R$.

To construct the actual projector, we need only build an operator which will assemble the removed boxes in the correct way to produce $s$. We will give an example of how to construct this operator; the general case should be clear. Lets start with the representation $R$ shown, removing the boxes indicated

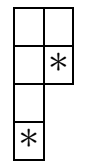

Our projector will act in the subspace spanned by the two sets of states

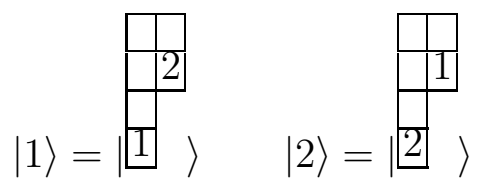

We are using a Young-Yamonouchi basis. Thus, each state given above could be any one of $d_{\boxplus}=3$ states, corresponding to the number of ways to complete the labels. When acting in the subspace, the operator which organizes the boxes into representation $s$ is

$$
P_{s}=\frac{d_{s}}{m !} \sum_{\sigma \in S_{m}} \chi_{s}(\sigma) \Gamma_{R}(\sigma) .
$$


All that remains is to supply a formula for the action of $\Gamma_{R}(\sigma)$ (for $\sigma=1,(12)$ ) when acting on the subspace spanned by $|1\rangle$ and $|2\rangle$. The action of $\Gamma_{R}(\sigma)$ on any YoungYamonouchi state is well known. For the states above

$$
\begin{gathered}
\Gamma_{R}((12))|1\rangle=-\frac{1}{3}|1\rangle+\frac{\sqrt{8}}{3}|2\rangle \\
\Gamma_{R}((12))|2\rangle=\frac{1}{3}|2\rangle+\frac{\sqrt{8}}{3}|1\rangle
\end{gathered}
$$

so that

$$
\Gamma_{R}((12))=-\frac{1}{3}|1\rangle\left\langle 1\left|+\frac{\sqrt{8}}{3}\right| 2\right\rangle\left\langle 1\left|+\frac{1}{3}\right| 2\right\rangle\left\langle 2\left|+\frac{\sqrt{8}}{3}\right| 1\right\rangle\langle 2|
$$

and

$$
\Gamma_{R}(1)=|1\rangle\langle 1|+| 2\rangle\langle 2|
$$

\section{The Radial Direction}

In this section we describe a limit in which the dilatation operator simplifies significantly. There are two columns in the Young diagrams labeling the restricted Schur polynomials. When the first column contains $O(\sqrt{N})$ boxes more than the second, the dilatation operator simplifies to a lattice realization of the second derivative. The Young diagram label itself defines the lattice.

\subsection{Three Impurities}

The three impurity operators are built using many $Z$ s and three $Y$ s. To specify these operators, we need to give the three Young diagrams labeling the restricted Schur polynomial. The second Young diagram, $r$, (which specifies an irreducible representation of $\left.S_{n}\right)$ is specified by stating the number of rows with two boxes $\left(=b_{0}\right)$ and the number of rows with a single box $\left(=b_{1}\right)$. The third Young diagram label, $s$, (which specifies an irreducible representation of $S_{3}$ ) and the first Young diagram label, $R$, (which specifies an irreducible representation of $S_{n+3}$ ) can now be built from $r$ by specifying which boxes in $R$ are to be removed to obtain $r$ and how these boxes are to be organized into an $S_{3}$ irreducible representation. There are 6 possibilities

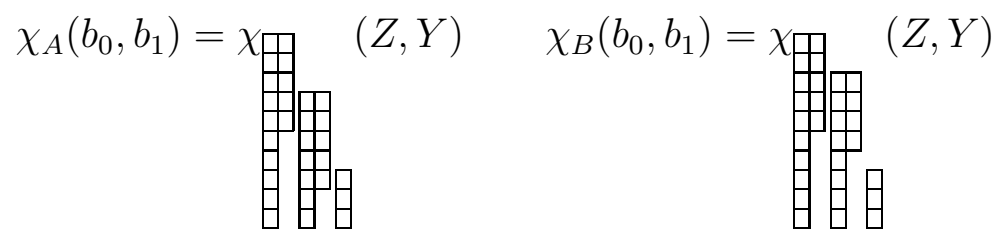






The corresponding normalized operators are denoted using the capital letter $O$. In view of the discussion of section 3 , we know that $b_{0}$ is $O(N)$ and $b_{1}$ ranges from 0 or 1 to $O(N)$. The action of the dilatation operator is given in Appendix A.

The $\mathcal{R}$-charge of an operator in the field theory maps into the angular momentum of the dual string theory state. Thanks to the Myers effect[51] the angular momentum of the string theory state determines its size. Identifying the two columns of the Young diagrams with the two threebranes, the number of boxes in each column determines the angular momentum and hence the size of each threebrane. In the limit that $N-b_{0}=$ $O(N), b_{0}=O(N)$ and $b_{1}=O(\sqrt{N})$ we have non-maximal giants which are separated by a distance of $O(1)$ in string units. In this limit, we expect the dynamics to simplify. Indeed, the system should be described by two D3 brane giant gravitons with open strings stretching between then. The action of the dilatation operator becomes

$$
\begin{aligned}
& D O_{A}\left(b_{0}, b_{1}\right)=g_{Y M}^{2}\left(N-b_{0}\right) \times O\left(\frac{1}{b_{1}}\right) \\
D O_{B}\left(b_{0}, b_{1}\right)= & -\frac{4}{3} g_{Y M}^{2}\left(N-b_{0}\right)\left[O_{B}\left(b_{0}+1, b_{1}-2\right)-2 O_{B}\left(b_{0}, b_{1}\right)+O_{B}\left(b_{0}-1, b_{1}+2\right)\right] \\
+ & \frac{2 \sqrt{2}}{3} g_{Y M}^{2}\left(N-b_{0}\right)\left[O_{C}\left(b_{0}+1, b_{1}-2\right)-2 O_{C}\left(b_{0}+1, b_{1}\right)+O_{C}\left(b_{0}-1, b_{1}+2\right)\right] \\
D O_{C}\left(b_{0}, b_{1}\right)= & \frac{2 \sqrt{2}}{3} g_{Y M}^{2}\left(N-b_{0}\right)\left[O_{B}\left(b_{0}+1, b_{1}-2\right)-2 O_{B}\left(b_{0}, b_{1}\right)+O_{B}\left(b_{0}-1, b_{1}+2\right)\right] \\
& -\frac{2}{3} g_{Y M}^{2}\left(N-b_{0}\right)\left[O_{C}\left(b_{0}+1, b_{1}-2\right)-2 O_{C}\left(b_{0}, b_{1}\right)+O_{C}\left(b_{0}-1, b_{1}+2\right)\right] \\
D O_{D}\left(b_{0}, b_{1}\right)= & -\frac{4}{3} g_{Y M}^{2}\left(N-b_{0}\right)\left[O_{D}\left(b_{0}+1, b_{1}-2\right)-2 O_{D}\left(b_{0}, b_{1}\right)+O_{D}\left(b_{0}-1, b_{1}+2\right)\right] \\
& +\frac{2 \sqrt{2}}{3} g_{Y M}^{2}\left(N-b_{0}\right)\left[O_{E}\left(b_{0}+1, b_{1}-2\right)-2 O_{E}\left(b_{0}, b_{1}\right)+O_{E}\left(b_{0}-1, b_{1}+2\right)\right]
\end{aligned}
$$




$$
\begin{gathered}
D O_{E}\left(b_{0}, b_{1}\right)=\frac{2 \sqrt{2}}{3} g_{Y M}^{2}\left(N-b_{0}\right)\left[O_{D}\left(b_{0}+1, b_{1}-2\right)-2 O_{D}\left(b_{0}, b_{1}\right)+O_{D}\left(b_{0}-1, b_{1}+2\right)\right] \\
-\frac{2}{3} g_{Y M}^{2}\left(N-b_{0}\right)\left[O_{E}\left(b_{0}+1, b_{1}-2\right)-2 O_{E}\left(b_{0}, b_{1}\right)+O_{E}\left(b_{0}-1, b_{1}+2\right)\right] \\
D O_{F}\left(b_{0}, b_{1}\right)=g_{Y M}^{2}\left(N-b_{0}\right) \times O\left(\frac{1}{b_{1}}\right)
\end{gathered}
$$

These results have a natural interpretation. Notice that there are four operators for which the $S_{m}$ representation is the totally antisymmetric representation. We will see that there are also four operators for which the corresponding states remain supersymmetric; this agreement between the number of operators for which the $S_{m}$ representation is the totally antisymmetric representation and the number of supersymmetric states, holds in general for the two giant system. Looking at the labels, it is natural to interpret $O_{A}\left(b_{0}, b_{1}\right)$ as a state in which we deform only the larger threebrane. Recall from section 3, that deforming a single threebrane gives us a supersymmetric state so it seems natural for $O_{A}\left(b_{0}, b_{1}\right)$ to remain supersymmetric. Similarly, $O_{F}\left(b_{0}, b_{1}\right)$ can be interpreted as a state in which we deform only the smaller threebrane and a similar comment can be made. The fact that the combinations $O_{B}\left(b_{0}, b_{1}\right)+\sqrt{2} O_{C}\left(b_{0}, b_{1}\right)$ and $O_{D}\left(b_{0}, b_{1}\right)+\sqrt{2} O_{E}\left(b_{0}, b_{1}\right)$ are annihilated by $D$ implies that there are another two supersymmetric ways to deform the pair of threebranes. Finally, notice that if we set $O_{B}\left(b_{0}, b_{1}\right)-O_{C}\left(b_{0}, b_{1}\right) / \sqrt{2} \equiv O_{B-C}\left(b_{0}, b_{1}\right)$ and $O_{D}\left(b_{0}, b_{1}\right)-O_{E}\left(b_{0}, b_{1}\right) / \sqrt{2} \equiv O_{D-E}\left(b_{0}, b_{1}\right)$ we have

$$
\begin{aligned}
& D O_{B-C}\left(b_{0}, b_{1}\right)=-2 g_{Y M}^{2}\left(N-b_{0}\right)\left[O_{B-C}\left(b_{0}+1, b_{1}-2\right)-2 O_{B-C}\left(b_{0}, b_{1}\right)+O_{B-C}\left(b_{0}-1, b_{1}+2\right)\right] \\
& D O_{D-E}\left(b_{0}, b_{1}\right)=-2 g_{Y M}^{2}\left(N-b_{0}\right)\left[O_{D-E}\left(b_{0}+1, b_{1}-2\right)-2 O_{D-E}\left(b_{0}, b_{1}\right)+O_{D-E}\left(b_{0}-1, b_{1}+2\right)\right]
\end{aligned}
$$

The right hand side again is a discretization of the second derivative. It is the Young diagram itself that is defining the lattice. After recalling that the number of boxes in each column sets the angular momentum and hence the radius ${ }^{4}$ of the corresponding threebrane, its clear that the radius of the giant graviton together with local physics in this radial direction has emerged.

\subsection{Four Impurities}

For the case of four impurities there are nine possible operators that we can define
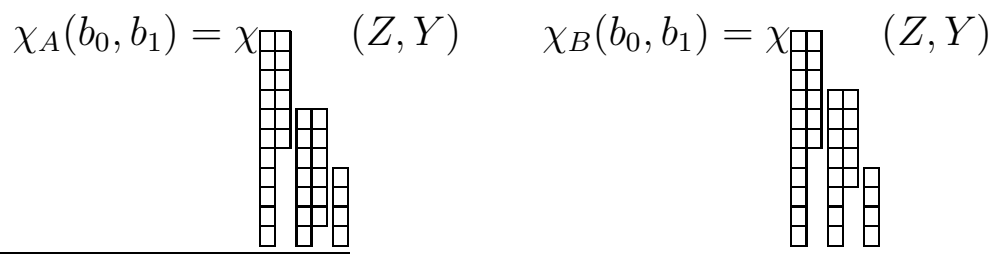

\footnotetext{
${ }^{4}$ The giant graviton threebrane wraps an $\mathrm{S}^{3}$ of a given radius. It is the radius of this $\mathrm{S}^{3}$ that we call the "radius of the threebrane".
} 


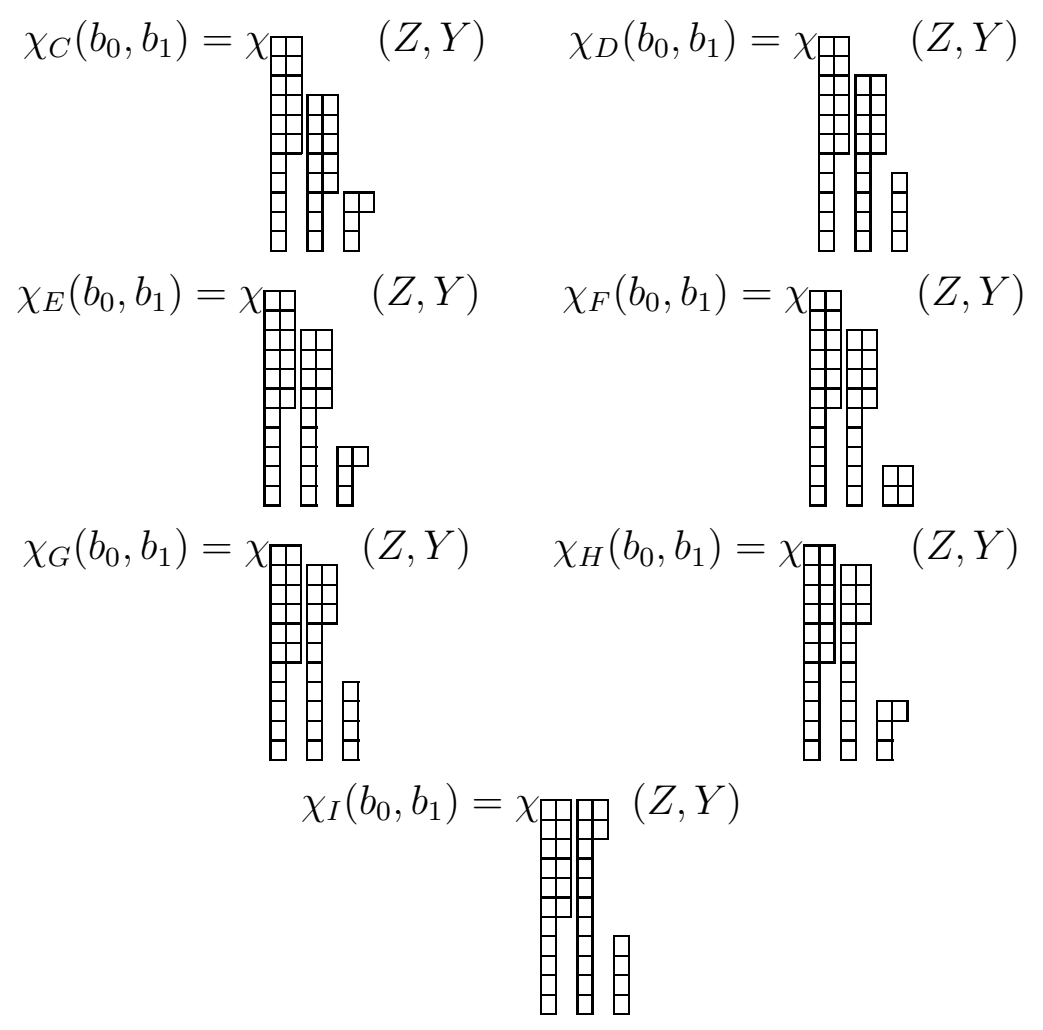

Again, the corresponding normalized operators are denoted using the capital letter $O$. The action of the dilatation operator is given in Appendix A.

In the limit that $N-b_{0}=O(N), b_{0}=O(N)$ and $b_{1}=O(\sqrt{N})$ the dynamics again simplifies. The action of the dilatation operator becomes

$$
\begin{aligned}
& D O_{A}\left(b_{0}, b_{1}\right)=\left(N-b_{0}\right) g_{Y M}^{2} \times O\left(\frac{1}{b_{1}}\right) \\
D O_{B}\left(b_{0}, b_{1}\right)= & -\frac{3}{2} g_{Y M}^{2}\left(N-b_{0}\right)\left[O_{B}\left(b_{0}+1, b_{1}-2\right)-2 O_{B}\left(b_{0}, b_{1}\right)+O_{B}\left(b_{0}-1, b_{1}+2\right)\right] \\
+ & \frac{\sqrt{3}}{2} g_{Y M}^{2}\left(N-b_{0}\right)\left[O_{C}\left(b_{0}+1, b_{1}-2\right)-2 O_{C}\left(b_{0}+1, b_{1}\right)+O_{C}\left(b_{0}-1, b_{1}+2\right)\right] \\
D O_{C}\left(b_{0}, b_{1}\right)= & \frac{\sqrt{3}}{2} g_{Y M}^{2}\left(N-b_{0}\right)\left[O_{B}\left(b_{0}+1, b_{1}-2\right)-2 O_{B}\left(b_{0}, b_{1}\right)+O_{B}\left(b_{0}-1, b_{1}+2\right)\right] \\
& -\frac{1}{2} g_{Y M}^{2}\left(N-b_{0}\right)\left[O_{C}\left(b_{0}+1, b_{1}-2\right)-2 O_{C}\left(b_{0}, b_{1}\right)+O_{C}\left(b_{0}-1, b_{1}+2\right)\right] \\
D O_{D}\left(b_{0}, b_{1}\right)= & -2 g_{Y M}^{2}\left(N-b_{0}\right)\left[O_{D}\left(b_{0}+1, b_{1}-2\right)-2 O_{D}\left(b_{0}, b_{1}\right)+O_{D}\left(b_{0}-1, b_{1}+2\right)\right] \\
& +\frac{2}{\sqrt{3}} g_{Y M}^{2}\left(N-b_{0}\right)\left[O_{E}\left(b_{0}+1, b_{1}-2\right)-2 O_{E}\left(b_{0}+1, b_{1}\right)+O_{E}\left(b_{0}-1, b_{1}+2\right)\right]
\end{aligned}
$$




$$
\begin{aligned}
D O_{E}\left(b_{0}, b_{1}\right)= & -2 g_{Y M}^{2}\left(N-b_{0}\right)\left[O_{E}\left(b_{0}+1, b_{1}-2\right)-2 O_{E}\left(b_{0}, b_{1}\right)+O_{E}\left(b_{0}-1, b_{1}+2\right)\right] \\
& +\frac{2}{\sqrt{3}} g_{Y M}^{2}\left(N-b_{0}\right)\left[O_{D}\left(b_{0}+1, b_{1}-2\right)-2 O_{D}\left(b_{0}, b_{1}\right)+O_{D}\left(b_{0}-1, b_{1}+2\right)\right] \\
& +\frac{2 \sqrt{6}}{3} g_{Y M}^{2}\left(N-b_{0}\right)\left[O_{F}\left(b_{0}+1, b_{1}-2\right)-2 O_{F}\left(b_{0}, b_{1}\right)+O_{F}\left(b_{0}-1, b_{1}+2\right)\right] \\
D O_{F}\left(b_{0}, b_{1}\right)= & -2 g_{Y M}^{2}\left(N-b_{0}\right)\left[O_{F}\left(b_{0}+1, b_{1}-2\right)-2 O_{F}\left(b_{0}, b_{1}\right)+O_{F}\left(b_{0}-1, b_{1}+2\right)\right] \\
& +\frac{2 \sqrt{6}}{3} g_{Y M}^{2}\left(N-b_{0}\right)\left[O_{E}\left(b_{0}+1, b_{1}-2\right)-2 O_{E}\left(b_{0}+1, b_{1}\right)+O_{E}\left(b_{0}-1, b_{1}+2\right)\right] \\
D O_{G}\left(b_{0}, b_{1}\right)= & -\frac{3}{2} g_{Y M}^{2}\left(N-b_{0}\right)\left[O_{G}\left(b_{0}+1, b_{1}-2\right)-2 O_{G}\left(b_{0}, b_{1}\right)+O_{G}\left(b_{0}-1, b_{1}+2\right)\right] \\
& +\frac{\sqrt{3}}{2} g_{Y M}^{2}\left(N-b_{0}\right)\left[O_{H}\left(b_{0}+1, b_{1}-2\right)-2 O_{H}\left(b_{0}+1, b_{1}\right)+O_{H}\left(b_{0}-1, b_{1}+2\right)\right] \\
& +\frac{1}{2} g_{Y M}^{2}\left(N-b_{0}\right)\left[O_{H}\left(b_{0}+1, b_{1}-2\right)-2 O_{H}\left(b_{0}, b_{1}\right)+O_{H}\left(b_{0}-1, b_{1}+2\right)\right] \\
& +\frac{\sqrt{3}}{2} g_{Y M}^{2}\left(N-b_{0}\right)\left[O_{G}\left(b_{0}+1, b_{1}-2\right)-2 O_{G}\left(b_{0}+1, b_{1}\right)+O_{G}\left(b_{0}-1, b_{1}+2\right)\right] \\
D O_{H}\left(b_{0}, b_{1}\right)= & D O_{I}\left(b_{0}, b_{1}\right)=\left(N-b_{0}\right) g_{Y M}^{2} \times O\left(\frac{1}{b_{1}}\right)
\end{aligned}
$$

We can again identify combinations of operators that are annihilated by $D$, that is, that are BPS. Apart from $O_{A}\left(b_{0}, b_{1}\right)$ and $O_{I}\left(b_{0}, b_{1}\right)$ we have $O_{B}\left(b_{0}, b_{1}\right)+\sqrt{3} O_{C}\left(b_{0}, b_{1}\right)$, $O_{D}\left(b_{0}, b_{1}\right)+\sqrt{3} O_{E}\left(b_{0}, b_{1}\right)+\sqrt{2} O_{F}\left(b_{0}, b_{1}\right)$ and $O_{G}\left(b_{0}, b_{1}\right)+\sqrt{3} O_{H}\left(b_{0}, b_{1}\right)$. Notice that all of the BPS operators from this section and the last can be written as

$$
O_{\mathrm{BPS}}(R, r)=\sum_{s} \sqrt{d_{s}} O_{R,(r, s)}\left(b_{0}, b_{1}\right)
$$

where $d_{s}$ is the dimension of the irreducible representation $s$ of the symmetric group. Finally, notice that if we set $\sqrt{3} O_{B}\left(b_{0}, b_{1}\right)-O_{C}\left(b_{0}, b_{1}\right) \equiv O_{B-C}\left(b_{0}, b_{1}\right), \sqrt{2} O_{D}\left(b_{0}, b_{1}\right)-$ $O_{F}\left(b_{0}, b_{1}\right) \equiv O_{D-F}\left(b_{0}, b_{1}\right), O_{D}\left(b_{0}, b_{1}\right)-\sqrt{3} O_{E}\left(b_{0}, b_{1}\right)+\sqrt{2} O_{F}\left(b_{0}, b_{1}\right) \equiv O_{D F-E}\left(b_{0}, b_{1}\right)$ and $\sqrt{3} O_{G}\left(b_{0}, b_{1}\right)-O_{H}\left(b_{0}, b_{1}\right) \equiv O_{G-H}\left(b_{0}, b_{1}\right)$, we have

$$
\begin{aligned}
D O_{B-C}\left(b_{0}, b_{1}\right) & =-2 g_{Y M}^{2}\left(N-b_{0}\right)\left[O_{B-C}\left(b_{0}+1, b_{1}-2\right)-2 O_{B-C}\left(b_{0}, b_{1}\right)+O_{B-C}\left(b_{0}-1, b_{1}+2\right)\right] \\
D O_{D-F}\left(b_{0}, b_{1}\right) & =-2 g_{Y M}^{2}\left(N-b_{0}\right)\left[O_{D-F}\left(b_{0}+1, b_{1}-2\right)-2 O_{D-F}\left(b_{0}, b_{1}\right)+O_{D-F}\left(b_{0}-1, b_{1}+2\right)\right] \\
D O_{D F-E}\left(b_{0}, b_{1}\right) & =-4 g_{Y M}^{2}\left(N-b_{0}\right)\left[O_{D F-E}\left(b_{0}+1, b_{1}-2\right)-2 O_{D F-E}\left(b_{0}, b_{1}\right)+O_{D F-E}\left(b_{0}-1, b_{1}+2\right)\right] \\
D O_{G-H}\left(b_{0}, b_{1}\right) & =-2 g_{Y M}^{2}\left(N-b_{0}\right)\left[O_{G-H}\left(b_{0}+1, b_{1}-2\right)-2 O_{G-H}\left(b_{0}, b_{1}\right)+O_{G-H}\left(b_{0}-1, b_{1}+2\right)\right]
\end{aligned}
$$

The right hand side is again a discretization of the second derivative. 


\section{Numerical Results}

In this section we describe the result of numerically diagonalizing the dilatation operator.

When setting up a numerical computation of the spectrum of the anomalous dimension operator, we need to specify the maximum value for the difference between the number of boxes in the long column and the number of boxes in the short column. Given this value, denoted $a_{\max }$, we are able to determine how many operators participate in our problem and we are able to describe the resulting spectrum rather explicitly. We will focus on the case of even $a_{\text {max }}$. In this case the difference between the number of boxes in the long column and the number of boxes in the short column is always an even number.

\subsection{Two Impurities}

For a given value of $a_{\max }$ there are $2+2 a_{\max }$ states in total. There are $\frac{3}{2} a_{\max }+1$ zero eigenvalues (corresponding to supersymmetric states). The remaining eigenvalues are

$$
\lambda_{i}=8 g_{Y M}^{2} i \quad i=1,2, \cdots, \frac{a_{\max }}{2}+1 .
$$

\subsection{Three Impurities}

For a given value of $a_{\max }$ there are $1+3 a_{\max }$ states in total. There are $2 a_{\max }$ zero eigenvalues (corresponding to supersymmetric states). The remaining eigenvalues are

$$
\lambda_{i}=8 g_{Y M}^{2} i \quad i=1,2, \cdots, \frac{a_{\max }}{2},
$$

each with a degeneracy of two and a single maximum eigenvalue $\lambda=4 a_{\max } g_{Y M}^{2}+8 g_{Y M}^{2}$. This degeneracy almost certainly indicates a symmetry enhancement in the large $N$ limit.

\subsection{Four Impurities}

For a given value of $a_{\max }$ there are $1+\frac{9}{2} a_{\max }$ states in total. There are $\frac{5}{2} a_{\max }-1$ zero eigenvalues. The eigenvalues are again evenly spaced with a level spacing of $8 g_{Y M}^{2}$ and they are again degenerate. The low lying eigenvalues

$$
\lambda_{i}=8 g_{Y M}^{2} i \quad i=1,2, \cdots, \frac{a_{\max }}{2},
$$

have a degeneracy which alternates between 3 and 4 . Thus, we find three eigenvalues $\lambda=8 g_{Y M}^{2}$, followed by four eigenvalues $\lambda=16 g_{Y M}^{2}$, followed by three eigenvalues $\lambda=24 g_{Y M}^{2}$, followed by four eigenvalues $\lambda=32 g_{Y M}^{2}$ and so on. 
If $a_{\max }$ is a multiple of 4 , the larger eigenvalues are given by $\lambda=4 a_{\max } g_{Y M}^{2}+8 g_{Y M}^{2}$, $\lambda=4 a_{\max } g_{Y M}^{2}+16 g_{Y M}^{2}$ and

$$
\lambda_{i}=4 a_{\max } g_{Y M}^{2}+16 g_{Y M}^{2}+16 i g_{Y M}^{2} \quad i=1,2, \cdots, \frac{a_{\max }}{4} ;
$$

all of these eigenvalues on the last line above are non-degenerate.

If $a_{\max }$ (which by assumption is even) is not a multiple of 4 , the larger eigenvalues are given by $\lambda=4 a_{\max } g_{Y M}^{2}+8 g_{Y M}^{2}$ with a degeneracy of 2 and

$$
\lambda_{i}=4 a_{\max } g_{Y M}^{2}+16 g_{Y M}^{2}+16 i g_{Y M}^{2} \quad i=1,2, \cdots, \frac{a_{\max }+2}{4} ;
$$

all of these eigenvalues on the last line above are non-degenerate.

Once again, the degeneracies observed almost certainly indicate a symmetry enhancement in the large $N$ limit.

\section{Discussion}

In this article we have computed the one loop anomalous dimension of an operator built from $O(N) Z$ s and 3 or $4 Y$ "impurities". What lessons can be learnt from these results, together with the results of [30], which dealt with the case of 2 impurities? Before we start the discussion, it is useful to recall the structure of the operators which participate in the case of two impurities

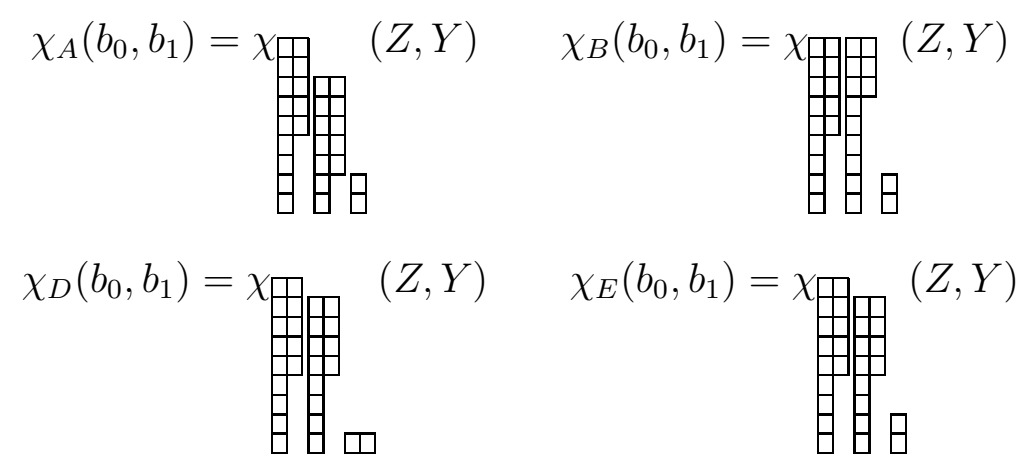

Firstly, the actual result for the one loop dilation operator (see Appendix A) is rather complicated. This is to be expected - it was obtained by summing a huge class of Feynman diagrams - much more than just the planar diagrams. On the other hand, the spectra of anomalous dimensions obtained are rather simple. To obtain a numerically tractable problem, we have been forced to keep the value of $a_{\max }$ finite (recall that this parameter measures the maximum value for the difference between the number of boxes in the long column and the number of boxes in the short column). In 
the large $N$ limit $^{5} a_{\max }=\zeta N$ goes to infinity. Our discussion assumes we are working in this $a_{\max } \rightarrow \infty$ limit.

We have restricted ourselves to a study of less than five impurities. This restriction is not imposed because our methods break down for five or more impurities, but only because the details of writing down the projection operators and evaluating the dilatation operator becomes increasingly complicated as the number of impurities is increased. In particular, there is no problem in principle with taking $O(N)$ impurities. The case of no impurities or one impurity are simple to handle analytically - all of these operators are annihilated by the one loop dilatation operator[30].

For the case of two impurities there are three times as many zero eigenvalue states as there positive eigenvalue states. There are $\frac{a_{\max }}{2}$ positive eigenvalue states with a constant energy level spacing of $8 g_{Y M}^{2}$. Thus, it is natural to associate an oscillator with a set of $\sim \frac{a_{\max }}{2}$ states. With this assumption, the dilatation operator acting on the two impurity operators gives the spectrum of three harmonic oscillators with a level spacing of zero and a single harmonic oscillator with a level spacing of $8 g_{Y M}^{2}$. Looking at the two impurity operators given above, we see that there are three operators with impurities in the antisymmetric representation $(\Theta)$ and one operator with the impurities in the symmetric representation (Ш).

For the case of three impurities, the dilatation operator gives the spectrum of four harmonic oscillators with a level spacing of zero and two harmonic oscillators with a level spacing of $8 g_{Y M}^{2}$; each oscillator again has $\sim \frac{a_{\max }}{2}$ states. Looking at the three impurity operators given in section 5.1 , we see that there are four operators $(A, B, D$ and $\mathrm{F}$ ) with impurities in the antisymmetric representation $(\mathrm{B})$ and two operators $(\mathrm{C}$ and E) with the impurities in the $\boxplus$ representation.

For the case of four impurities, recall that we had an interesting degeneracy structure - the degeneracy alternates between three degenerate states and four degenerate states. This is naturally explained as three oscillators with a level spacing of $8 g_{Y M}^{2}$ and a fourth with a level spacing of $16 g_{Y M}^{2}$; with this interpretation each oscillator again has $\sim \frac{a_{\max }}{2}$ states. Thus, for the case of four impurities, the dilatation operator gives the spectrum of five harmonic oscillators with a level spacing of zero, three harmonic oscillator with a level spacing of $8 g_{Y M}^{2}$ and one with a spacing of $16 g_{Y M}^{2}$. Looking at the four impurity operators given in section 5.2, we see that there are five operators $(A, B, D, G$ and I) with impurities in the antisymmetric representation $(B)$, three operators $(\mathrm{C}, \mathrm{E}$ and $\mathrm{H})$ with the impurities in the $\mathrm{G}$ representation and one $(\mathrm{F})$ with

\footnotetext{
${ }^{5} \zeta$ was defined in section 3.
} 
impurities in the $\boxplus$ representation ${ }^{6}$.

It is rather easy to guess the result for a general number of impurities. If the number of impurities is even $=2 n$ we expect to obtain a set of oscillators with frequency $\omega_{i}$ and degeneracy $d_{i}$ given by

$$
\omega_{i}=8 i g_{Y M}^{2}, \quad d_{i}=2(n-i)+1, \quad i=0,1, \ldots, n .
$$

If the number of impurities is odd $=2 n+1$ we expect to obtain a set of oscillators with frequency $\omega_{i}$ and degeneracy $d_{i}$ given by

$$
\omega_{i}=8 i g_{Y M}^{2}, \quad d_{i}=2(n-i+1), \quad i=0,1, \ldots, n .
$$

This conjecture passes a simple counting test: $\sum_{i} d_{i}$ is equal to the number of restricted Schur polynomials that can be defined. Further, the degeneracies $d_{i}$ match the number of each type of oscillator that can be defined: $d_{i}$ is equal to the number of operators which have the impurities organized into a Young diagram with $i$ boxes in the short column.

A beautiful simple picture is emerging from the rather complicated formulas obtained for the dilatation operator: the dilatation operator is equivalent to a set of harmonic oscillators. For each type of operator there is a single oscillator and the frequency of the oscillator is determined by the representation which organizes the impurities. Since a set of harmonic oscillators is an integrable system, this system we have studied here is an example of an integrable dilatation operator, obtained by summing planar and non-planar diagrams.

$\mathcal{N}=4$ super Yang-Mills theory has an $S U(4) \mathcal{R}$-symmetry. A $U(2)$ subgroup of the $\mathcal{R}$-symmetry can be used to rotate the $Y$ s and $Z$ s into each other. Thus, although the operators we have studied do not treat the $Y$ s and $Z$ s symmetrically, a more complete study working with the complete set of two column restricted Schur polynomials would yield a description in which $Z$ and $Y$ appear on an equal footing. If we are to recover this symmetric description its clear that the oscillators which emerge for each particular impurity configuration must themselves have frequencies which are multiples of $8 g_{Y M}^{2}$ - exactly as we have found. Exploring this further we should be able to show that the spectrum is filling out $U(2)$ multiplets. By including more species of impurities we should also be able to see more of the expected global symmetry group.

What is the AdS/CFT dual interpretation of our results? The operators we have considered are dual to giant gravitons. A connection between the geometry of giant

\footnotetext{
${ }^{6}$ Note that by looking at the representation that organizes the impurities we have been able to read off the frequencies of the harmonic oscillators appearing. This is where it ends; in particular we are not claiming that $O_{F}$ are the operators corresponding to the frequency $16 g_{Y M}^{2}$ operators! Operators with a good scaling dimension are a complicated linear combination of the various possible $O$ s.
} 
gravitons and harmonic oscillators was already uncovered in [34, 35, 36]. This work quantizes the moduli space of Mikhailov's giant gravitons. Consequently one is capturing a huge space of states. It is this huge space of states that connects to harmonic oscillators. Our study has focused on a two giant system. Consequently, we know that the oscillators that we have captured are associated to this two giant system and excitations of it. We have thus arrived at a slightly more refined statement of how the harmonic oscillator enters. The critical reader might question whether our set of operators includes excitations corresponding (for example) to the two giant system plus a graviton. This would seem to be a small perturbation of the two giant system that is not an excitation of it - the graviton is an excitation of spacetime. We do not have such excitations among our states: these states correspond to operators with a small third column, which have decoupled at large $N$. In much the same way that by quantizing the possible excitation modes of a string one obtains a set of oscillators, its natural to think that our oscillators arise from the quantization of the possible excitation modes of a giant graviton.

We have described a limit (the first column of the Young diagram contains $O(\sqrt{N})$ boxes more than the second) in which the dilatation operator simplifies to a lattice realization of the second derivative. It is the Young diagram itself that is defining the lattice. After recalling that the number of boxes in each column sets the angular momentum and hence the radius of the corresponding threebrane, its clear that the radius of the giant graviton together with local physics in this radial direction has emerged. Notice that for BMN loops the number of lattice sites is $O(\sqrt{N})$; for the operators we have studied here the number of lattice sites is $O(N)$.

One can contemplate a number of tests for our proposal. With a thorough understanding of the vibrational modes of the giant graviton system, one could imagine realizing definite classical membrane geometries by considering coherent states of the oscillators. One might then compare the energy of these states with the volume of the membrane times the membrane tension. Although naively the field theory and gravity computations are never simultaneously valid, one might hope that for operators which are close to BPS, the perturbative result might agree with the strong coupling answer (see [39] for a relevant discussion).

In addition to questions we pointed out above, there are a number of clear directions for further study. Given the simplicity of our results, it should be possible to construct an analytic solution. This is under investigation[52]. It would also be interesting to understand how our results are modified at higher loops. One could also consider the case of $n>2$ column restricted Schur polynomials and more species of impurities. A much more general question would be to ask when (and how) simple systems are expected to emerge from multimatrix models. For a single matrix model, it is well 
known that the planar limit is nicely captured by the dynamics of $N$ non-interacting non-relativistic fermions in an external potential. In this paper we have argued that the large $N$ limit of a class of operators dual to giant gravitons is captured by a collection of harmonic oscillators. Presumably every semiclassical object in spacetime (string, giant graviton, black hole, etc) is associated with the emergence of a simple system in the large $N$ limit of the corresponding class of operators in the field theory. Can we make this connection sharper and more useful?

Acknowledgements: We would like to thank Tom Brown, Warren Carlson, Norman Ives, Yusuke Kimura, Hai Lin, Dimitri Polyakov, Sanjaye Ramgoolam, Peter Roenne, Stephanie Smith and Michael Stephanou for pleasant discussions and/or helpful correspondence. This work is based upon research supported by the South African Research Chairs Initiative of the Department of Science and Technology and National Research Foundation. Any opinion, findings and conclusions or recommendations expressed in this material are those of the authors and therefore the NRF and DST do not accept any liability with regard thereto.

\section{A. Dilatation Operator for Three or Four impurities}

The dilatation operator for the case of two impurities has been given in [30]. In what follows

$$
D O=g_{Y M}^{2} \hat{D} O
$$

\section{A.1 Three Impurities}

$$
\begin{aligned}
& \hat{D} O_{A}\left(b_{0}, b_{1}\right)=\sqrt{\left(N-b_{0}-b_{1}-2\right)\left(N-b_{0}+1\right)}\left[4 b_{1} \sqrt{\frac{b_{1}+4}{b_{1}+2}} \frac{1}{\left(b_{1}+2\right)\left(b_{1}+3\right)} O_{B}\left(b_{0}, b_{1}\right)\right. \\
& -2 \sqrt{\frac{b_{1}+4}{b_{1}+2} \sqrt{2}} \frac{1}{\left(b_{1}+2\right)} O_{C}\left(b_{0}, b_{1}\right)+8 \sqrt{\frac{\left(b_{1}+4\right)\left(b_{1}+1\right)}{\left(b_{1}+2\right)\left(b_{1}+3\right)}} \frac{1}{\left(b_{1}+3\right)\left(b_{1}+2\right)} O_{D}\left(b_{0}-1, b_{1}+2\right) \\
& \left.+2 \sqrt{\frac{\left(b_{1}+4\right)\left(b_{1}+1\right)}{\left(b_{1}+3\right)\left(b_{1}+2\right)}} \frac{\sqrt{2}}{\left(b_{1}+3\right)\left(b_{1}+2\right)} O_{E}\left(b_{0}-1, b_{1}+2\right)\right]+\left(N-b_{0}-b_{1}-2\right)\left[\frac{12}{\left(b_{1}+2\right)\left(b_{1}+3\right)} O_{A}\left(b_{0}, b_{1}\right)\right. \\
& \left.-4 \frac{\sqrt{\left(b_{1}+1\right)\left(b_{1}+3\right)}\left(b_{1}+5\right)}{\left(b_{1}+3\right)^{2}\left(b_{1}+2\right)} O_{B}\left(b_{0}-1, b_{1}+2\right)+2 \frac{\sqrt{\left(b_{1}+1\right)\left(b_{1}+3\right)} \sqrt{2}}{\left(b_{1}+3\right)^{2}} O_{C}\left(b_{0}-1, b_{1}+2\right)\right]
\end{aligned}
$$




$$
\begin{aligned}
& \hat{D} O_{B}\left(b_{0}, b_{1}\right)=\sqrt{\left(N-b_{0}-b_{1}-1\right)\left(N-b_{0}\right)}\left[-\frac{4}{3} \sqrt{\frac{\left(b_{1}+2\right)\left(b_{1}-1\right)}{b_{1}\left(b_{1}+1\right)}} \frac{\left(b_{1}-2\right)\left(b_{1}+3\right)}{b_{1}\left(b_{1}+1\right)} O_{B}\left(b_{0}+1, b_{1}-2\right)\right. \\
& +\frac{2}{3} \frac{b_{1}+3}{b 1} \sqrt{\frac{\left(b_{1}+2\right)\left(b_{1}-1\right)}{\left(b_{1}+1\right) b_{1}}} \sqrt{2} O_{C}\left(b_{0}+1, b_{1}-2\right)-\frac{32}{3} \frac{b_{1}{ }^{2}+2 b_{1}-3}{b_{1}\left(b_{1}+1\right)\left(b_{1}+2\right)^{2}} \sqrt{\frac{b_{1}+2}{b_{1}}} O_{D}\left(b_{0}, b_{1}\right) \\
& \left.-\frac{2 \sqrt{2}}{3} \sqrt{\frac{b_{1}+2}{b_{1}}} \frac{\left(b_{1}+3\right)\left(3 b_{1}-2\right)}{b_{1}\left(b_{1}+2\right)\left(b_{1}+1\right)} O_{E}\left(b_{0}, b_{1}\right)+8 \sqrt{\frac{\left(b_{1}+3\right) b_{1}}{\left(b_{1}+2\right)\left(b_{1}+1\right)}} \frac{1}{\left(b_{1}+1\right)\left(b_{1}+2\right)} O_{F}\left(b_{0}-1, b_{1}+2\right)\right] \\
& +\sqrt{\left(N-b_{0}-b_{1}-2\right)\left(N-b_{0}+1\right)}\left[\frac{2}{3} \sqrt{\frac{\left(b_{1}+4\right)\left(b_{1}+1\right)}{\left(b_{1}+2\right)\left(b_{1}+3\right)}} \frac{\sqrt{2} b_{1}}{\left(b_{1}+3\right)} O_{C}\left(b_{0}-1, b_{1}+2\right)\right. \\
& -\frac{4}{3} \sqrt{\frac{\left(b_{1}+4\right)\left(b_{1}+1\right)}{\left(b_{1}+3\right)\left(b_{1}+2\right)}} \frac{\left(b_{1}+5\right) b_{1}}{\left(b_{1}+3\right)\left(b_{1}+2\right)} O_{B}\left(b_{0}-1, b_{1}+2\right) \\
& \left.+4 \sqrt{\frac{b_{1}+4}{b_{1}+2}} \frac{b_{1}}{\left(b_{1}+3\right)\left(b_{1}+2\right)} O_{A}\left(b_{0}, b_{1}\right)\right]+\left(N-b_{0}-b_{1}-1\right)\left[-4 \sqrt{\frac{b_{1}-1}{b_{1}+1}} \frac{\left(b_{1}+3\right)}{\left(b_{1}+1\right) b_{1}} O_{A}\left(b_{0}+1, b_{1}-2\right)\right. \\
& +\frac{4}{3} \frac{\left(b_{1}+3\right)\left(b_{1}^{3}+5 b_{1}^{2}+8 b_{1}-12\right)}{\left(b_{1}+1\right) b_{1}\left(b_{1}+2\right)^{2}} O_{B}\left(b_{0}, b_{1}\right)-\frac{2 \sqrt{2}}{3} \frac{\left(b_{1}^{2}+2 b_{1}-4\right)\left(b_{1}+3\right)}{\left(b_{1}+1\right)\left(b_{1}+2\right)^{2}} O_{C}\left(b_{0}, b_{1}\right) \\
& \left.-\frac{8}{3} \sqrt{\frac{b_{1}+3}{b_{1}+1}} \frac{\left(b_{1}+4\right) b_{1}}{\left(b_{1}+2\right)^{2}\left(b_{1}+1\right)} O_{D}\left(b_{0}-1, b_{1}+2\right)+\frac{4}{3} \sqrt{2} \sqrt{\frac{b_{1}+3}{b_{1}+1}} \frac{b_{1}}{\left(b_{1}+2\right)^{2}} O_{E}\left(b_{0}-1, b_{1}+2\right)\right] \\
& +\left(N-b_{0}+1\right)\left[\frac{4}{3} \frac{\left(b_{1}+4\right) b_{1}^{2}}{\left(b_{1}+3\right)\left(b_{1}+2\right)^{2}} O_{B}\left(b_{0}, b_{1}\right)+\frac{8}{3} \frac{\sqrt{\left(b_{1}+1\right)\left(b_{1}+3\right)} b_{1}\left(b_{1}+4\right)}{\left(b_{1}+3\right)^{2}\left(b_{1}+2\right)^{2}} O_{D}\left(b_{0}-1, b_{1}+2\right)\right. \\
& \left.-\frac{2}{3} \frac{\sqrt{2}\left(b_{1}+4\right) b_{1}}{\left(b_{1}+2\right)^{2}} O_{C}\left(b_{0}, b_{1}\right)+\frac{2}{3} \frac{\sqrt{2} \sqrt{\left(b_{1}+1\right)\left(b_{1}+3\right)} b_{1}\left(b_{1}+4\right)}{\left(b_{1}+3\right)^{2}\left(b_{1}+2\right)^{2}} O_{E}\left(b_{0}-1, b_{1}+2\right)\right]
\end{aligned}
$$$$
\hat{D} O_{C}\left(b_{0}, b_{1}\right)=\sqrt{\left(N-b_{0}-b_{1}-1\right)\left(N-b_{0}\right)}\left[\frac{2 \sqrt{2}}{3} \sqrt{\frac{\left(b_{1}+2\right)\left(b_{1}-1\right)}{\left(b_{1}+1\right) b_{1}}} \frac{\left(b_{1}-2\right)}{b_{1}+1} O_{B}\left(b_{0}+1, b_{1}-2\right)\right.
$$$$
\begin{aligned}
& -\frac{2}{3} \sqrt{\frac{\left(b_{1}+2\right)\left(b_{1}-1\right)}{\left(b_{1}+1\right) b_{1}}} O_{C}\left(b_{0}+1, b_{1}-2\right)+\frac{2 \sqrt{2}}{3} \sqrt{\frac{b_{1}+2}{b_{1}}} \frac{\left(b_{1}-1\right)\left(3 b_{1}+8\right)}{\left(b_{1}+1\right)\left(b_{1}+2\right)^{2}} O_{D}\left(b_{0}, b_{1}\right) \\
& \left.-\frac{4}{3} \sqrt{\frac{b_{1}+2}{b_{1}}} \frac{1}{\left(b_{1}+1\right)\left(b_{1}+2\right)} O_{E}\left(b_{0}, b_{1}\right)+2 \frac{\sqrt{\left(b_{1}+2\right)\left(b_{1}+3\right)\left(b_{1}+1\right) b_{1}} \sqrt{2}}{\left(b_{1}+1\right)^{2}\left(b_{1}+2\right)^{2}} O_{F}\left(b_{0}-1, b_{1}+2\right)\right]
\end{aligned}
$$ 


$$
\begin{gathered}
+\sqrt{\left(N-b_{0}-b_{1}-2\right)\left(N-b_{0}+1\right)}\left[-2 \frac{\sqrt{\left(b_{1}+4\right)\left(b_{1}+2\right)} \sqrt{2}}{\left(b_{1}+2\right)^{2}} O_{A}\left(b_{0}, b_{1}\right)\right. \\
\left.+\frac{2 \sqrt{2}}{3} \sqrt{\frac{\left(b_{1}+4\right)\left(b_{1}+1\right)}{\left(b_{1}+3\right)\left(b_{1}+2\right)}} \frac{\left(b_{1}+5\right)}{\left(b_{1}+2\right)} O_{B}\left(b_{0}-1, b_{1}+2\right)-\frac{2}{3} \sqrt{\frac{\left(b_{1}+4\right)\left(b_{1}+1\right)}{\left(b_{1}+2\right)\left(b_{1}+3\right)}} O_{C}\left(b_{0}-1, b_{1}+2\right)\right] \\
+\left(N-b_{0}-b_{1}-1\right)\left[2 \sqrt{\frac{b_{1}-1}{b_{1}+1}} \sqrt{2} \frac{1}{b_{1}+1} O_{A}\left(b_{0}+1, b_{1}-2\right)\right. \\
-\frac{2}{3} \sqrt{2} \sqrt{\left.\frac{b_{1}+3}{b_{1}+1} \frac{\left(b_{1}+4\right)}{\left(b_{1}+2\right)^{2}\left(b_{1}+1\right)} b_{D}\left(b_{0}-1, b_{1}+2\right)+\frac{2}{3} \sqrt{\frac{b_{1}+3}{b_{1}+1}} \frac{b_{1}}{\left(b_{1}+2\right)^{2}} O_{E}\left(b_{0}-1, b_{1}+2\right)\right]} \\
+\left(N-b_{0}+1\right)\left[-\frac{2}{3} \frac{\sqrt{2}\left(b_{1}+4\right) b_{1}}{\left(b_{1}+2\right)^{2}} O_{B}\left(b_{0}, b_{1}\right)+\frac{2}{3} \frac{\left(b_{1}+4\right)\left(b_{1}+3\right)}{\left(b_{1}+2\right)^{2}} O_{C}\left(b_{0}, b_{1}\right)\right. \\
-\frac{4}{3} \frac{\sqrt{2} \sqrt{\left(b_{1}+1\right)\left(b_{1}+3\right)}\left(b_{1}+4\right)}{\left(b_{1}+3\right)\left(b_{1}+2\right)^{2}} O_{D}\left(b_{0}-1, b_{1}\right)+\frac{2}{3} \frac{b_{1}\left(b_{1}^{2}+2 b_{1}-1\right)}{\left(b_{1}+1\right)\left(b_{1}+2\right)^{2}} O_{C}\left(b_{0}, b_{1}\right)
\end{gathered}
$$$$
\hat{D} O_{D}\left(b_{0}, b_{1}\right)=\sqrt{\left(N-b_{0}-b_{1}\right)\left(N-b_{0}-1\right)}\left[-\frac{4}{3} \sqrt{\frac{\left(b_{1}+1\right)\left(b_{1}-2\right)}{b_{1}\left(b_{1}-1\right)}} \frac{\left(b_{1}-3\right)\left(b_{1}+2\right)}{b_{1}\left(b_{1}-1\right)} O_{D}\left(b_{0}+1, b_{1}-2\right)\right.
$$$$
\left.+\frac{2}{3} \frac{\left(b_{1}+2\right) \sqrt{b_{1}\left(b_{1}-1\right)\left(b_{1}+1\right)\left(b_{1}-2\right)} \sqrt{2}}{b_{1}\left(b_{1}-1\right)^{2}} O_{E}\left(b_{0}+1, b_{1}-2\right)-4 \frac{\left(b_{1}+2\right) \sqrt{b_{1}\left(b_{1}-2\right)}}{b_{1}{ }^{2}\left(b_{1}-1\right)} O_{F}\left(b_{0}, b_{1}\right)\right]
$$$$
+\sqrt{\left(N-b_{0}-b_{1}-1\right)\left(N-b_{0}\right)}\left[\frac{8}{b_{1}\left(b_{1}+1\right)} \sqrt{\frac{\left(b_{1}+2\right)\left(b_{1}-1\right)}{\left(b_{1}+1\right) b_{1}}} O_{A}\left(b_{0}+1, b_{1}-2\right)\right.
$$$$
+\frac{2 \sqrt{2}}{3} \frac{\sqrt{b_{1}\left(b_{1}+2\right)}\left(b_{1}-1\right)\left(3 b_{1}+8\right)}{\left(b_{1}+1\right)\left(b_{1}+2\right)^{2} b_{1}} O_{C}\left(b_{0}, b_{1}\right)-\frac{32}{3} \frac{\left(b_{1}^{2}+2 b_{1}-3\right) \sqrt{b_{1}\left(b_{1}+2\right)}}{\left(b_{1}+2\right)^{2} b_{1}^{2}\left(b_{1}+1\right)} O_{B}\left(b_{0}, b_{1}\right)
$$$$
-\frac{4}{3} \sqrt{\frac{b_{1}\left(b_{1}+3\right)}{\left(b_{1}+2\right)\left(b_{1}+1\right)}} \frac{\left(b_{1}-1\right)\left(b_{1}+4\right)}{\left(b_{1}+2\right)\left(b_{1}+1\right)} O_{D}\left(b_{0}-1, b_{1}+2\right)
$$$$
\left.+\frac{2}{3} \sqrt{\frac{b_{1}\left(b_{1}+3\right)}{\left(b_{1}+2\right)\left(b_{1}+1\right)}} \sqrt{2} \frac{\left(b_{1}-1\right)}{b_{1}+2} O_{E}\left(b_{0}-1, b_{1}+2\right)\right]+\left(N-b_{0}\right)\left[\frac{4}{3} \frac{\left(b_{1}-1\right)\left(b_{1}^{3}+b_{1}^{2}+16\right)}{\left(b_{1}+1\right) b_{1}^{2}\left(b_{1}+2\right)} O_{D}\left(b_{0}, b_{1}\right)\right.
$$$$
+\frac{8}{3} \sqrt{\frac{b_{1}-1}{b_{1}+1}} \frac{b_{1}^{2}-4}{b_{1}^{2}\left(b_{1}+1\right)} O_{B}\left(b_{0}+1, b_{1}-2\right)-\frac{4}{3} \sqrt{2} \sqrt{\frac{b_{1}-1}{b_{1}+1}} \frac{b_{1}+2}{b_{1}^{2}} O_{C}\left(b_{0}+1, b_{1}-2\right)
$$ 


$$
\begin{aligned}
& \left.-\frac{2 \sqrt{2}}{3} \frac{\left(b_{1}^{2}+2 b_{1}-4\right)\left(b_{1}-1\right)}{\left(b_{1}+1\right) b_{1}^{2}} O_{E}\left(b_{0}, b_{1}\right)+4\left(b_{1}-1\right) \sqrt{\frac{b_{1}+3}{b_{1}+1}} \frac{1}{\left(b_{1}+2\right)\left(b_{1}+1\right)} O_{F}\left(b_{0}-1, b_{1}+2\right)\right] \\
& +\left(N-b_{0}-b_{1}\right)\left[\frac{4}{3} \frac{\left(b_{1}-2\right)\left(b_{1}+2\right)^{2}}{\left(b_{1}-1\right) b_{1}^{2}} O_{D}\left(b_{0}, b_{1}\right)-\frac{2 \sqrt{2}}{3} \frac{\left(b_{1}^{2}-4\right)}{b_{1}^{2}} O_{E}\left(b_{0}, b_{1}\right)\right. \\
& \left.-\frac{8}{3} \sqrt{\frac{\left(b_{1}+1\right)}{\left(b_{1}-1\right)}} \frac{\left(b_{1}^{2}-4\right)}{b_{1}^{2}\left(b_{1}-1\right)} O_{B}\left(b_{0}+1, b_{1}-2\right)-\frac{2 \sqrt{2}}{3} \sqrt{\frac{\left(b_{1}+1\right)}{\left(b_{1}-1\right)}} \frac{\left(b_{1}^{2}-4\right)}{b_{1}^{2}\left(b_{1}-1\right)} O_{C}\left(b_{0}+1, b_{1}-2\right)\right]
\end{aligned}
$$$$
\hat{D} O_{E}\left(b_{0}, b_{1}\right)=\sqrt{\left(N-b_{0}-b_{1}\right)\left(N-b_{0}-1\right)}\left[\frac{2 \sqrt{2}}{3} \sqrt{\frac{\left(b_{1}+1\right)\left(b_{1}-2\right)}{b_{1}\left(b_{1}-1\right)}} \frac{\left(b_{1}-3\right)}{b_{1}} O_{D}\left(b_{0}+1, b_{1}-2\right)\right.
$$$$
\left.-\frac{2}{3} \frac{\sqrt{b_{1}\left(b_{1}-1\right)\left(b_{1}+1\right)\left(b_{1}-2\right)}}{b_{1}\left(b_{1}-1\right)} O_{E}\left(b_{0}+1, b_{1}-2\right)+2 \frac{\sqrt{2} \sqrt{b_{1}\left(b_{1}-2\right)}}{b_{1}{ }^{2}} O_{F}\left(b_{0}, b_{1}\right)\right]
$$$$
+\sqrt{\left(N-b_{0}-b_{1}-1\right)\left(N-b_{0}\right)}\left[2 \frac{\sqrt{2} \sqrt{\left(b_{1}+2\right)\left(b_{1}-1\right)\left(b_{1}+1\right) b_{1}}}{b_{1}^{2}\left(b_{1}+1\right)^{2}} O_{A}\left(b_{0}+1, b_{1}-2\right)\right.
$$$$
-\frac{4}{3} \frac{\sqrt{b_{1}\left(b_{1}+2\right)}}{\left(b_{1}+2\right)\left(b_{1}+1\right) b_{1}} O_{C}\left(b_{0}, b_{1}\right)-\frac{2 \sqrt{2}}{3} \sqrt{b_{1}\left(b_{1}+2\right)} \frac{\left(b_{1}+3\right)\left(3 b_{1}-2\right)}{\left(b_{1}+1\right) b_{1}^{2}\left(b_{1}+2\right)} O_{B}\left(b_{0}, b_{1}\right)
$$$$
\left.+\frac{2}{3} \sqrt{\frac{b_{1}\left(b_{1}+3\right)}{\left(b_{1}+2\right)\left(b_{1}+1\right)}} \sqrt{2} \frac{b_{1}+4}{b_{1}+1} O_{D}\left(b_{0}-1, b_{1}+2\right)-\frac{2}{3} \sqrt{\frac{b_{1}\left(b_{1}+3\right)}{\left(b_{1}+1\right)\left(b_{1}+2\right)}} O_{E}\left(b_{0}-1, b_{1}+2\right)\right]
$$$$
+\left(N-b_{0}\right)\left[-\frac{2 \sqrt{2}}{3} \frac{\left(b_{1}^{2}+2 b_{1}-4\right)\left(b_{1}-1\right)}{\left(b_{1}+1\right) b_{1}^{2}} O_{D}\left(b_{0}, b_{1}\right)\right.
$$$$
+\frac{2}{3} \frac{\left(b_{1}+2\right)\left(b_{1}^{2}+2 b_{1}-1\right)}{\left(b_{1}+1\right) b_{1}^{2}} O_{E}\left(b_{0}, b_{1}\right)-2 \sqrt{\frac{b_{1}+3}{b_{1}+1}} \sqrt{2} \frac{1}{b_{1}+1} O_{F}\left(b_{0}-1, b_{1}+2\right)
$$$$
\left.+\frac{2}{3} \sqrt{2} \sqrt{\frac{b_{1}-1}{b_{1}+1}} \frac{b_{1}^{2}-4}{\left(b_{1}+1\right) b_{1}^{2}} O_{B}\left(b_{0}+1, b_{1}-2\right)-\frac{2}{3} \sqrt{\frac{b_{1}-1}{b_{1}+1}} \frac{b_{1}+2}{b_{1}^{2}} O_{C}\left(b_{0}+1, b_{1}-2\right)\right]
$$$$
+\left(N-b_{0}-b_{1}\right)\left[\frac{2}{3} \frac{\left(b_{1}^{2}-3 b_{1}+2\right)}{b_{1}{ }^{2}} O_{E}\left(b_{0}, b_{1}\right)-\frac{2 \sqrt{2}}{3} \frac{\left(b_{1}^{2}-4\right)}{b_{1}^{2}} O_{D}\left(b_{0}, b_{1}\right)\right.
$$

$$
\left.+\frac{4 \sqrt{2}}{3} \frac{\left(b_{1}-2\right) \sqrt{\left(b_{1}+1\right)\left(b_{1}-1\right)}}{{b_{1}{ }^{2}\left(b_{1}-1\right)}_{b_{B}}} O_{B}\left(b_{0}+1, b_{1}-2\right)+\frac{2}{3} \frac{\left(b_{1}-2\right) \sqrt{\left(b_{1}+1\right)\left(b_{1}-1\right)}}{b_{1}{ }^{2}\left(b_{1}-1\right)} O_{C}\left(b_{0}+1, b_{1}-2\right)\right]
$$

$$
\hat{D} O_{F}\left(b_{0}, b_{1}\right)=\sqrt{\left(N-b_{0}-b_{1}\right)\left(N-b_{0}-1\right)}\left[8 \frac{\sqrt{b_{1}\left(b_{1}-1\right)\left(b_{1}+1\right)\left(b_{1}-2\right)}}{b_{1}^{2}\left(b_{1}-1\right)^{2}} O_{B}\left(b_{0}+1, b_{1}-2\right)\right.
$$




$$
\begin{gathered}
+2 \frac{\sqrt{2} \sqrt{b_{1}\left(b_{1}-1\right)\left(b_{1}+1\right)\left(b_{1}-2\right)}}{b_{1}^{2}\left(b_{1}-1\right)^{2}} O_{C}\left(b_{0}+1, b_{1}-2\right)-4\left(b_{1}+2\right) \sqrt{\frac{b_{1}-2}{b_{1}}} \frac{1}{b_{1}\left(b_{1}-1\right)} O_{D}\left(b_{0}, b_{1}\right) \\
\left.+2 \sqrt{\frac{b_{1}-2}{b_{1}}} \sqrt{2} \frac{1}{b_{1}} O_{E}\left(b_{0}, b_{1}\right)\right]+4 \frac{\left(b_{1}-3\right) \sqrt{\left(b_{1}+1\right)\left(b_{1}-1\right)}\left(N-b_{0}-1\right)}{b_{1}\left(b_{1}-1\right)^{2}} O_{D}\left(b_{0}+1, b_{1}-2\right) \\
-2 \frac{\sqrt{\left(b_{1}+1\right)\left(b_{1}-1\right)} \sqrt{2}\left(N-b_{0}-1\right)}{\left(b_{1}-1\right)^{2}} O_{E}\left(b_{0}+1, b_{1}-2\right)+12 \frac{N-b_{0}-1}{b_{1}\left(b_{1}-1\right)} O_{F}\left(b_{0}, b_{1}\right)
\end{gathered}
$$

\section{A.2 Four Impurities}

$$
\begin{gathered}
\hat{D} O_{A}\left(b_{0}, b_{1}\right)=\sqrt{\left(N-b_{0}-b_{1}-3\right)\left(N-b_{0}+1\right)}\left[6 \sqrt{\frac{b_{1}+5}{b_{1}+3}} \frac{b_{1}}{\left(b_{1}+4\right)\left(b_{1}+2\right)} O_{B}\left(b_{0}, b_{1}\right)\right. \\
-2 \sqrt{3} \sqrt{\frac{b_{1}+5}{b_{1}+3}} \frac{1}{b_{1}+2} O_{C}\left(b_{0}, b_{1}\right)+12 \frac{\sqrt{\left(b_{1}+1\right)\left(b_{1}+5\right)}}{\left(b_{1}+3\right)\left(b_{1}+2\right)\left(b_{1}+4\right)} O_{D}\left(b_{0}-1, b_{1}+2\right) \\
\left.+4 \sqrt{3} \frac{\sqrt{\left(b_{1}+5\right)\left(b_{1}+1\right)}}{\left(b_{1}+3\right)\left(b_{1}+4\right)\left(b_{1}+2\right)} O_{E}\left(b_{0}-1, b_{1}+2\right)\right]+\left(N-b_{0}-b_{1}-3\right)\left[\frac{24}{\left(b_{1}+2\right)\left(b_{1}+4\right)} O_{A}\left(b_{0}, b_{1}\right)\right. \\
\left.-6 \sqrt{\frac{b_{1}+1}{b_{1}+3}} \frac{b_{1}+6}{\left(b_{1}+2\right)\left(b_{1}+4\right)} O_{B}\left(b_{0}-1, b_{1}+2\right)+2 \sqrt{3} \sqrt{\frac{b_{1}+1}{b_{1}+3}} \frac{1}{b_{1}+4} O_{C}\left(b_{0}-1, b_{1}+2\right)\right]
\end{gathered}
$$

$$
\begin{aligned}
& \hat{D} O_{B}\left(b_{0}, b_{1}\right)=\sqrt{\left(N-b_{0}-b_{1}-2\right)\left(N-b_{0}\right)}\left[\frac{\sqrt{3}}{2} \sqrt{\left(b_{1}+3\right)\left(b_{1}-1\right)} \frac{b_{1}+4}{b_{1}\left(b_{1}+1\right)} O_{C}\left(b_{0}+1, b_{1}-2\right)\right. \\
& -\frac{3}{2} \frac{\sqrt{\left(b_{1}+3\right)\left(b_{1}-1\right)}}{\left(b_{1}+1\right)\left(b_{1}+2\right)} \frac{\left(b_{1}+4\right)\left(b_{1}-2\right)}{b_{1}} O_{B}\left(b_{0}+1, b_{1}-2\right)+3 \frac{\left(b_{1}+4\right)\left(b_{1}-1\right)\left(b_{1}-6\right)}{b_{1}\left(b_{1}+3\right)\left(b_{1}+2\right)^{2}} \sqrt{\frac{b_{1}+3}{b_{1}+1}} O_{D}\left(b_{0}, b_{1}\right) \\
& \left.-\sqrt{3} \frac{\left(b_{1}+4\right)\left(3 b_{1}-2\right)}{b_{1}\left(b_{1}+2\right)^{2}} \sqrt{\frac{b_{1}+3}{b_{1}+1}} O_{E}\left(b_{0}, b_{1}\right)+\frac{2 b_{1}\left(b_{1}+4\right)}{\left(b_{1}+1\right)\left(b_{1}+3\right)\left(b_{1}+2\right)^{2}}\left(9 O_{G}\left(b_{0}-1, b_{1}+2\right)+\sqrt{3} O_{H}\left(b_{0}-1, b_{1}+2\right)\right)\right] \\
& +\sqrt{\left(N-b_{0}-b_{1}-3\right)\left(N-b_{0}+1\right)}\left[-\frac{3}{2} \sqrt{\left(b_{1}+5\right)\left(b_{1}+1\right)} \frac{b_{1}\left(b_{1}+6\right)}{\left(b_{1}+3\right)\left(b_{1}+2\right)\left(b_{1}+4\right)} O_{B}\left(b_{0}-1, b_{1}+2\right)\right. \\
& \left.+\frac{\sqrt{3}}{2} \sqrt{\left(b_{1}+5\right)\left(b_{1}+1\right)} \frac{b_{1}}{\left(b_{1}+3\right)\left(b_{1}+4\right)} O_{C}\left(b_{0}-1, b_{1}+2\right)+6 \sqrt{\left(b_{1}+5\right)\left(b_{1}+3\right)} \frac{b_{1}}{\left(b_{1}+2\right)\left(b_{1}+3\right)\left(b_{1}+4\right)} O_{A}\left(b_{0}, b_{1}\right)\right] \\
& +\left(N-b_{0}-b_{1}-2\right)\left[-6 \sqrt{\frac{b_{1}-1}{b_{1}+1}} \frac{b_{1}+4}{b_{1}\left(b_{1}+2\right)} O_{A}\left(b_{0}+1, b_{1}-2\right)+\frac{3}{2} \frac{\left(b_{1}^{3}+7 b_{1}^{2}+22 b_{1}-24\right)\left(b_{1}+4\right)}{b_{1}\left(b_{1}+2\right)^{2}\left(b_{1}+3\right)} O_{B}\left(b_{0}, b_{1}\right)\right. \\
& -\frac{\sqrt{3}}{2} \frac{\left(b_{1}^{2}+3 b_{1}-6\right)\left(b_{1}+4\right)}{\left(b_{1}+2\right)^{2}\left(b_{1}+3\right)} O_{C}\left(b_{0}, b_{1}\right)-6 \sqrt{\frac{b_{1}+3}{b_{1}+1}} \frac{b_{1}\left(b_{1}+5\right)\left(b_{1}+4\right)}{\left(b_{1}+2\right)^{2}\left(b_{1}+3\right)^{2}} O_{D}\left(b_{0}-1, b_{1}+2\right) \\
& \left.+\sqrt{12} \sqrt{\left(b_{1}+3\right)\left(b_{1}+1\right)} \frac{b_{1}\left(b_{1}+4\right)}{\left(b_{1}+3\right)^{2}\left(b_{1}+2\right)^{2}} O_{E}\left(b_{0}-1, b_{1}+2\right)\right]+\left(N-b_{0}+1\right)\left[\frac{3}{2} \frac{b_{1}^{2}\left(b_{1}+5\right)}{\left(b_{1}+2\right)\left(b_{1}+3\right)\left(b_{1}+4\right)} O_{B}\left(b_{0}, b_{1}\right)\right.
\end{aligned}
$$




$$
\begin{gathered}
-\frac{\sqrt{3}}{2} \frac{\left(b_{1}+5\right) b_{1}}{\left(b_{1}+2\right)\left(b_{1}+3\right)} O_{C}\left(b_{0}, b_{1}\right)+3 \sqrt{\left(b_{1}+3\right)\left(b_{1}+1\right)} \frac{b_{1}\left(b_{1}+5\right)}{\left(b_{1}+3\right)^{2}\left(b_{1}+4\right)\left(b_{1}+2\right)} O_{D}\left(b_{0}-1, b_{1}+2\right) \\
\left.+\sqrt{3} \sqrt{\left(b_{1}+3\right)\left(b_{1}+1\right)} \frac{b_{1}\left(b_{1}+5\right)}{\left(b_{1}+3\right)^{2}\left(b_{1}+2\right)\left(b_{1}+4\right)} O_{E}\left(b_{0}-1, b_{1}+2\right)\right]
\end{gathered}
$$

$\hat{D} O_{C}\left(b_{0}, b_{1}\right)=\sqrt{\left(N-b_{0}-b_{1}-2\right)\left(N-b_{0}\right)}\left[\frac{\sqrt{3}}{2} \sqrt{\left(b_{1}-1\right)\left(b_{1}+3\right)} \frac{b_{1}-2}{\left(b_{1}+1\right)\left(b_{1}+2\right)} O_{B}\left(b_{0}+1, b_{1}-2\right)\right.$

$$
\begin{aligned}
& -\frac{1}{2} \sqrt{\left(b_{1}+3\right)\left(b_{1}-1\right)} \frac{1}{b_{1}+1} O_{C}\left(b_{0}+1, b_{1}-2\right)+\frac{1}{\sqrt{3}} \sqrt{\frac{b_{1}+3}{b_{1}+1}} \frac{\left(b_{1}-1\right)\left(5 b_{1}+18\right)}{\left(b_{1}+3\right)\left(b_{1}+2\right)^{2}} O_{D}\left(b_{0}, b_{1}\right) \\
& +\sqrt{\frac{b_{1}+3}{b_{1}+1}} \frac{3 b_{1}-2}{\left(b_{1}+2\right)^{2}} O_{E}\left(b_{0}, b_{1}\right)+2 \sqrt{3} \frac{b_{1}\left(b_{1}+4\right)}{\left(b_{1}+1\right)\left(b_{1}+2\right)^{2}\left(b_{1}+3\right)} O_{G}\left(b_{0}-1, b_{1}+2\right) \\
& \left.-\frac{4 \sqrt{6}}{3} \sqrt{\frac{b_{1}+3}{b_{1}+1}} \frac{1}{b_{1}+2} O_{F}\left(b_{0}, b_{1}\right)+2 \frac{\left(3 b_{1}^{2}+12 b_{1}+8\right)}{\left(b_{1}+3\right)\left(b_{1}+1\right)\left(b_{1}+2\right)^{2}} O_{H}\left(b_{0}-1, b_{1}+2\right)\right] \\
& +\sqrt{\left(N-b_{0}-b_{1}-3\right)\left(N-b_{0}+1\right)}\left[\frac{\sqrt{3}}{2} \sqrt{\left(b_{1}+5\right)\left(b_{1}+1\right)} \frac{b_{1}+6}{\left(b_{1}+3\right)\left(b_{1}+2\right)} O_{B}\left(b_{0}-1, b_{1}+2\right)\right. \\
& \left.-\frac{1}{2} \frac{\sqrt{\left(b_{1}+5\right)\left(b_{1}+1\right)}}{\left(b_{1}+3\right)} O_{C}\left(b_{0}-1, b_{1}+2\right)-2 \sqrt{3} \frac{\sqrt{\left(b_{1}+5\right)\left(b_{1}+3\right)}}{\left(b_{1}+3\right)\left(b_{1}+2\right)} O_{A}\left(b_{0}, b_{1}\right)\right]+\left(N-b_{0}-b_{1}-2\right) \times \\
& \times\left[\sqrt{\frac{b_{1}-1}{b_{1}+1}} \frac{2 \sqrt{3}}{b_{1}+2} O_{A}\left(b_{0}+1, b_{1}-2\right)-\frac{\sqrt{3}}{2} \frac{\left(b_{1}^{2}+3 b_{1}-6\right)\left(b_{1}+4\right)}{\left(b_{1}+2\right)^{2}\left(b_{1}+3\right)} O_{B}\left(b_{0}, b_{1}\right)+\frac{b_{1}^{3}+3 b_{1}^{2}+10 b_{1}+32}{2\left(b_{1}+2\right)^{2}\left(b_{1}+3\right)} O_{C}\left(b_{0}, b_{1}\right)\right. \\
& -\frac{2}{\sqrt{3}} \sqrt{\frac{b_{1}+3}{b_{1}+1}} \frac{b_{1}\left(b_{1}+5\right)\left(b_{1}+4\right)}{\left(b_{1}+2\right)^{2}\left(b_{1}+3\right)^{2}} O_{D}\left(b_{0}-1, b_{1}+2\right)-2 \sqrt{\left(b_{1}+3\right)\left(b_{1}+1\right)} \frac{\left(b_{1}+4\right)^{2}}{\left(b_{1}+2\right)^{2}\left(b_{1}+3\right)^{2}} O_{E}\left(b_{0}-1, b_{1}+2\right) \\
& \left.+\frac{4 \sqrt{2}}{\sqrt{3}} \frac{\sqrt{\left(b_{1}+3\right)\left(b_{1}+1\right)}}{\left(b_{1}+2\right)\left(b_{1}+3\right)} O_{F}\left(b_{0}-1, b_{1}+2\right)\right]+\left(N-b_{0}+1\right)\left[-\frac{\sqrt{3}}{2} \frac{b_{1}\left(b_{1}+5\right)}{\left(b_{1}+2\right)\left(b_{1}+3\right)} O_{B}\left(b_{0}, b_{1}\right)\right. \\
& +\frac{1}{2} \frac{\left(b_{1}+5\right)\left(b_{1}+4\right)}{\left(b_{1}+2\right)\left(b_{1}+3\right)} O_{C}\left(b_{0}, b_{1}\right)-\sqrt{3} \sqrt{\left(b_{1}+3\right)\left(b_{1}+1\right)} \frac{b_{1}+5}{\left(b_{1}+3\right)^{2}\left(b_{1}+2\right)} O_{D}\left(b_{0}-1, b_{1}+2\right) \\
& \left.-\sqrt{\left(b_{1}+3\right)\left(b_{1}+1\right)} \frac{\left(b_{1}+5\right)}{\left(b_{1}+2\right)\left(b_{1}+3\right)^{2}} O_{E}\left(b_{0}-1, b_{1}+2\right)\right] \\
& \hat{D} O_{D}\left(b_{0}, b_{1}\right)=\sqrt{\left(N-b_{0}-b_{1}-1\right)\left(N-b_{0}-1\right)}\left[-2 \frac{\left(b_{1}^{2}-9\right)\left(b_{1}^{2}-4\right)}{b_{1}^{2}\left(b_{1}^{2}-1\right)} O_{D}\left(b_{0}+1, b_{1}-2\right)\right. \\
& \left.+\frac{\left(b_{1}^{2}-4\right)\left(b_{1}+3\right)}{b_{1}^{2}\left(b_{1}+1\right)^{2}}\left[\frac{2\left(b_{1}+1\right)^{2}}{\sqrt{3}\left(b_{1}-1\right)} O_{E}\left(b_{0}+1, b_{1}-2\right)-\sqrt{\frac{b_{1}+1}{b_{1}-1}}\left(6 O_{G}\left(b_{0}, b_{1}\right)+\frac{2}{\sqrt{3}} O_{H}\left(b_{0}, b_{1}\right)\right)\right]\right]
\end{aligned}
$$




$$
\begin{aligned}
& +3 \sqrt{\frac{b_{1}-1}{b_{1}+1}} \frac{b_{1}{ }^{2}+b_{1}-6}{b_{1}\left(b_{1}+1\right)\left(b_{1}+2\right)} O_{G}\left(b_{0}, b_{1}\right)-\sqrt{3} \sqrt{\frac{b_{1}-1}{b_{1}+1}} \frac{b_{1}+3}{b_{1}\left(b_{1}+1\right)} O_{H}\left(b_{0}, b_{1}\right) \\
& \left.+12 \frac{\sqrt{\left(b_{1}+3\right)\left(b_{1}-1\right)}}{b_{1}\left(b_{1}+1\right)\left(b_{1}+2\right)} O_{I}\left(b_{0}-1, b_{1}+2\right)\right]+\sqrt{\left(N-b_{0}-b_{1}-2\right)\left(N-b_{0}\right)} \times \\
& \times\left[-3 \sqrt{\left(b_{1}+3\right)\left(b_{1}+1\right)} \frac{\left(b_{1}+4\right)\left(b_{1}-1\right)}{\left(b_{1}+1\right)^{2} b_{1}\left(b_{1}+2\right)} O_{B}\left(b_{0}, b_{1}\right)+\sqrt{3} \sqrt{\left(b_{1}+3\right)\left(b_{1}+1\right)} \frac{b_{1}-1}{\left(b_{1}+1\right)^{2}\left(b_{1}+2\right)} O_{C}\left(b_{0}, b_{1}\right)\right. \\
& +12 \frac{\sqrt{\left(b_{1}+3\right)\left(b_{1}-1\right)}}{b_{1}\left(b_{1}+1\right)\left(b_{1}+2\right)} O_{A}\left(b_{0}+1, b_{1}-2\right)-2 \frac{b_{1}\left(b_{1}-1\right)\left(b_{1}+4\right)\left(b_{1}+5\right)}{\left(b_{1}+1\right)\left(b_{1}+3\right)\left(b_{1}+2\right)^{2}} O_{D}\left(b_{0}-1, b_{1}+2\right) \\
& +6 \sqrt{\frac{b_{1}+1}{b_{1}+3}} \frac{b_{1}\left(b_{1}^{2}+3 b_{1}-4\right)}{\left(b_{1}+2\right)^{2}\left(b_{1}+1\right)^{2}} O_{B}\left(b_{0}, b_{1}\right)+\frac{2}{\sqrt{3}} \sqrt{\frac{b_{1}+1}{b_{1}+3}} \frac{b_{1}\left(b_{1}^{2}+3 b_{1}-4\right)}{\left(b_{1}+2\right)^{2}\left(b_{1}+1\right)^{2}} O_{C}\left(b_{0}, b_{1}\right) \\
& \left.+\frac{2}{\sqrt{3}} \frac{b_{1}\left(b_{1}+4\right)\left(b_{1}-1\right)}{\left(b_{1}+3\right)\left(b_{1}+2\right)^{2}} O_{E}\left(b_{0}-1, b_{1}+2\right)\right]+\left(N-b_{0}-b_{1}-1\right)\left[-6 \sqrt{\frac{b_{1}-1}{b_{1}+1}} \frac{b_{1}^{3}+3 b_{1}^{2}-4 b_{1}-12}{b_{1}^{2}\left(b_{1}^{2}-1\right)} O_{B}\left(b_{0}+1, b_{1}-2\right)\right. \\
& -\frac{2}{\sqrt{3}} \sqrt{\frac{b_{1}-1}{b_{1}+1}} \frac{b_{1}^{3}+3 b_{1}^{2}-4 b_{1}-12}{b_{1}^{2}\left(b_{1}^{2}-1\right)} O_{C}\left(b_{0}+1, b_{1}-2\right)+2 \frac{\left(b_{1}-2\right)\left(b_{1}+3\right)^{2}\left(b_{1}+2\right)}{b_{1}^{2}\left(b_{1}+1\right)^{2}} O_{D}\left(b_{0}, b_{1}\right) \\
& \left.-\frac{2}{\sqrt{3}} \frac{\left(b_{1}-2\right)\left(b_{1}+3\right)\left(b_{1}-1\right)\left(b_{1}+2\right)}{b_{1}^{2}\left(b_{1}+1\right)^{2}} O_{E}\left(b_{0}, b_{1}\right)\right]+\left(N-b_{0}\right)\left[3 \sqrt{\frac{b_{1}-1}{b_{1}+1}} \frac{b_{1}^{2}+b_{1}-6}{b_{1}\left(b_{1}+1\right)\left(b_{1}+2\right)} O_{B}\left(b_{0}+1, b_{1}-2\right)\right. \\
& -\sqrt{3} \sqrt{\frac{b_{1}-1}{b_{1}+1}} \frac{b_{1}+3}{b_{1}\left(b_{1}+1\right)} O_{C}\left(b_{0}+1, b_{1}-2\right)+6 \frac{\left(b_{1}+3\right)\left(b_{1}-1\right)}{b_{1}\left(b_{1}+2\right)\left(b_{1}+1\right)^{2}} O_{D}\left(b_{0}, b_{1}\right) \\
& \left.+2 \sqrt{3} \frac{\left(b_{1}+3\right)\left(b_{1}-1\right)}{\left(b_{1}+2\right) b_{1}\left(b_{1}+1\right)^{2}} O_{E}\left(b_{0}, b_{1}\right)\right]+\left(N-b_{0}-b_{1}-1\right)\left[6 \frac{\left(b_{1}+3\right)\left(b_{1}-1\right)}{\left(b_{1}+1\right)^{2} b_{1}\left(b_{1}+2\right)} O_{D}\left(b_{0}, b_{1}\right)\right. \\
& +2 \sqrt{3} \frac{\left(b_{1}-1\right)\left(b_{1}+3\right)}{\left(b_{1}+1\right)^{2} b_{1}\left(b_{1}+2\right)} O_{E}\left(b_{0}, b_{1}\right)-3 \sqrt{\frac{b_{1}+3}{b_{1}+1}} \frac{b_{1}^{2}+3 b_{1}-4}{b_{1}\left(b_{1}+1\right)\left(b_{1}+2\right)} O_{G}\left(b_{0}-1, b_{1}+2\right) \\
& \left.+\sqrt{3} \sqrt{\frac{b_{1}+3}{b_{1}+1}} \frac{\left(b_{1}-1\right)}{\left(b_{1}+1\right)\left(b_{1}+2\right)} O_{H}\left(b_{0}-1, b_{1}+2\right)\right]+\left(N-b_{0}\right)\left[2 \frac{\left(b_{1}+4\right)\left(b_{1}-1\right)^{2} b_{1}}{\left(b_{1}+1\right)^{2}\left(b_{1}+2\right)^{2}} O_{D}\left(b_{0}, b_{1}\right)\right. \\
& -\frac{2}{\sqrt{3}} \frac{\left(b_{1}-1\right)\left(b_{1}+3\right) b_{1}\left(b_{1}+4\right)}{\left(b_{1}+1\right)^{2}\left(b_{1}+2\right)^{2}} O_{E}\left(b_{0}, b_{1}\right)+6 \sqrt{\frac{b_{1}+3}{b_{1}+1}} \frac{\left(b_{1}^{2}+3 b_{1}-4\right) b_{1}}{\left(b_{1}+1\right)\left(b_{1}+2\right)^{2}\left(b_{1}+3\right)} O_{G}\left(b_{0}-1, b_{1}+2\right) \\
& \left.+\frac{2}{\sqrt{3}} \sqrt{\frac{b_{1}+3}{b_{1}+1}} \frac{\left(b_{1}^{2}+3 b_{1}-4\right) b_{1}}{\left(b_{1}+1\right)\left(b_{1}+2\right)^{2}\left(b_{1}+3\right)} O_{H}\left(b_{0}-1, b_{1}+2\right)\right] \\
& \hat{D} O_{E}\left(b_{0}, b_{1}\right)=\sqrt{\left(N-b_{0}-b_{1}-1\right)\left(N-b_{0}-1\right)}\left[\frac{2}{\sqrt{3}} \frac{b_{1}^{3}-3 b_{1}^{2}-4 b_{1}+12}{\left(b_{1}+1\right) b_{1}^{2}} O_{D}\left(b_{0}+1, b_{1}-2\right)\right. \\
& -2 \frac{\left(b_{1}^{2}-4\right)}{b_{1}^{2}} O_{E}\left(b_{0}+1, b_{1}-2\right)+\frac{2 \sqrt{6}}{3} \frac{b_{1}+2}{b_{1}} O_{F}\left(b_{0}+1, b_{1}-2\right)+\sqrt{3} \sqrt{\frac{b_{1}-1}{b_{1}+1}} \frac{\left(b_{1}-2\right)\left(3 b_{1}+8\right)}{b_{1}^{2}\left(b_{1}+2\right)} O_{G}\left(b_{0}, b_{1}\right) \\
& \left.-\sqrt{\frac{b_{1}-1}{b_{1}+1}} \frac{3 b_{1}+8}{b_{1}^{2}} O_{H}\left(b_{0}, b_{1}\right)+4 \sqrt{3} \frac{\sqrt{\left(b_{1}+3\right)\left(b_{1}-1\right)}}{b_{1}\left(b_{1}+1\right)\left(b_{1}+2\right)} O_{I}\left(b_{0}-1, b_{1}+2\right)\right]
\end{aligned}
$$




$$
\begin{aligned}
& +\sqrt{\left(N-b_{0}-b_{1}-2\right)\left(N-b_{0}\right)}\left[-\sqrt{3} \sqrt{\left(b_{1}+1\right)\left(b_{1}+3\right)} \frac{\left(b_{1}+4\right)\left(b_{1}-1\right)}{b_{1}\left(b_{1}+1\right)^{2}\left(b_{1}+2\right)} O_{B}\left(b_{0}, b_{1}\right)\right. \\
& +\sqrt{\left(b_{1}+1\right)\left(b_{1}+3\right)} \frac{b_{1}-1}{\left(b_{1}+2\right)\left(b_{1}+1\right)^{2}} O_{C}\left(b_{0}, b_{1}\right)+4 \sqrt{3} \frac{\sqrt{\left(b_{1}-1\right)\left(b_{1}+3\right)}}{b_{1}\left(b_{1}+1\right)\left(b_{1}+2\right)} O_{A}\left(b_{0}+1, b_{1}-2\right) \\
& -2 \sqrt{3} \sqrt{\left(b_{1}+3\right)\left(b_{1}+1\right)} \frac{b_{1}\left(b_{1}+4\right)}{\left(b_{1}+1\right)^{2}\left(b_{1}+2\right)^{2}} O_{B}\left(b_{0}, b_{1}\right) \\
& +2 \sqrt{\left(b_{1}+1\right)\left(b_{1}+3\right)} \frac{b_{1}^{2}}{\left(b_{1}+2\right)^{2}\left(b_{1}+1\right)^{2}} O_{C}\left(b_{0}, b_{1}\right)+\frac{2}{\sqrt{3}} \frac{b_{1}\left(b_{1}^{2}+9 b_{1}+20\right)}{\left(b_{1}+1\right)\left(b_{1}+2\right)^{2}} O_{D}\left(b_{0}-1, b_{1}+2\right) \\
& \left.-2 \frac{b_{1}\left(b_{1}+4\right)}{\left(b_{1}+2\right)^{2}} O_{E}\left(b_{0}-1, b_{1}+2\right)+\frac{2 \sqrt{2}}{\sqrt{3}} \frac{b_{1}}{\left(b_{1}+2\right)} O_{F}\left(b_{0}-1, b_{1}+2\right)\right]+\left(N-b_{0}-b_{1}-1\right) \times \\
& \times\left[2 \sqrt{3} \sqrt{\frac{b_{1}-1}{b_{1}+1}} \frac{b_{1}^{2}-4}{b_{1}^{2}\left(b_{1}+1\right)} O_{B}\left(b_{0}+1, b_{1}-2\right)-2 \sqrt{\frac{b_{1}-1}{b_{1}+1}} \frac{\left(b_{1}+2\right)^{2}}{b_{1}^{2}\left(b_{1}+1\right)} O_{C}\left(b_{0}+1, b_{1}-2\right)\right. \\
& -\frac{2}{\sqrt{3}} \frac{\left(b_{1}-2\right)\left(b_{1}+3\right)\left(b_{1}-1\right)\left(b_{1}+2\right)}{b_{1}^{2}\left(b_{1}+1\right)^{2}} O_{D}\left(b_{0}, b_{1}\right)+2 \frac{\left(b_{1}^{4}+2 b_{1}^{3}+b_{1}^{2}-4\right)}{b_{1}^{2}\left(b_{1}+1\right)^{2}} O_{E}\left(b_{0}, b_{1}\right) \\
& \left.-\frac{2 \sqrt{6}}{3} \frac{b_{1}^{2}+b_{1}-2}{b_{1}\left(b_{1}+1\right)} O_{F}\left(b_{0}, b_{1}\right)\right]+\left(N-b_{0}\right)\left[\sqrt{3} \sqrt{\frac{b_{1}-1}{b_{1}+1}} \frac{b_{1}^{2}+b_{1}-6}{\left(b_{1}+2\right) b_{1}\left(b_{1}+1\right)} O_{B}\left(b_{0}+1, b_{1}-2\right)\right. \\
& -\sqrt{\frac{b_{1}-1}{b_{1}+1}} \frac{b_{1}+3}{b_{1}\left(b_{1}+1\right)} O_{C}\left(b_{0}+1, b_{1}-2\right)+2 \sqrt{3} \frac{\left(b_{1}+3\right)\left(b_{1}-1\right)}{b_{1}\left(b_{1}+2\right)\left(b_{1}+1\right)^{2}} O_{D}\left(b_{0}, b_{1}\right) \\
& \left.+2 \frac{\left(b_{1}+3\right)\left(b_{1}-1\right)}{b_{1}\left(b_{1}+2\right)\left(b_{1}+1\right)^{2}} O_{E}\left(b_{0}, b_{1}\right)\right]+\left(N-b_{0}-b_{1}-1\right)\left[2 \sqrt{3} \frac{\left(b_{1}-1\right)\left(b_{1}+3\right)}{\left(b_{1}+1\right)^{2}\left(b_{1}+2\right) b_{1}} O_{D}\left(b_{0}, b_{1}\right)\right. \\
& +2 \frac{\left(b_{1}+3\right)\left(b_{1}-1\right)}{\left(b_{1}+1\right)^{2} b_{1}\left(b_{1}+2\right)} O_{E}\left(b_{0}, b_{1}\right)-\sqrt{3} \sqrt{\frac{b_{1}+3}{b_{1}+1}} \frac{b_{1}^{2}+3 b_{1}-4}{b_{1}\left(b_{1}+1\right)\left(b_{1}+2\right)} O_{G}\left(b_{0}-1, b_{1}+2\right) \\
& \left.+\sqrt{\frac{b_{1}+3}{b_{1}+1}} \frac{b_{1}-1}{\left(b_{1}+1\right)\left(b_{1}+2\right)} O_{H}\left(b_{0}-1, b_{1}+2\right)\right]+\left(N-b_{0}\right)\left[-\frac{2}{\sqrt{3}} \frac{\left(b_{1}-1\right)\left(b_{1}+3\right) b_{1}\left(b_{1}+4\right)}{\left(b_{1}+1\right)^{2}\left(b_{1}+2\right)^{2}} O_{D}\left(b_{0}, b_{1}\right)\right. \\
& +2 \frac{\left(b_{1}+3\right)\left(b_{1}^{2}+3 b_{1}+4\right) b_{1}}{\left(b_{1}+1\right)^{2}\left(b_{1}+2\right)^{2}} O_{E}\left(b_{0}, b_{1}\right)-\frac{2 \sqrt{6}}{3} \frac{\left(b_{1}+3\right) b_{1}}{\left(b_{1}+2\right)\left(b_{1}+1\right)} O_{F}\left(b_{0}, b_{1}\right) \\
& \left.-2 \sqrt{3} \sqrt{\frac{b_{1}+3}{b_{1}+1}} \frac{\left(b_{1}+4\right) b_{1}}{\left(b_{1}+1\right)\left(b_{1}+2\right)^{2}} O_{G}\left(b_{0}-1, b_{1}+2\right)+2 \sqrt{\frac{b_{1}+3}{b_{1}+1}} \frac{b_{1}^{2}}{\left(b_{1}+2\right)^{2}\left(b_{1}+1\right)} O_{H}\left(b_{0}-1, b_{1}+2\right)\right]
\end{aligned}
$$

$$
\begin{gathered}
\hat{D} O_{F}\left(b_{0}, b_{1}\right)=\sqrt{\left(N-b_{0}-b_{1}-1\right)\left(N-b_{0}-1\right)}\left[\frac{2 \sqrt{6}}{3} \frac{b_{1}-2}{b_{1}} O_{E}\left(b_{0}+1, b_{1}-2\right)-2 O_{F}\left(b_{0}+1, b_{1}-2\right)\right. \\
\left.+\frac{4 \sqrt{6}}{3} \frac{\sqrt{\left(b_{1}-1\right)\left(b_{1}+1\right)}}{b_{1}\left(b_{1}+1\right)} O_{H}\left(b_{0}, b_{1}\right)\right]+\sqrt{\left(N-b_{0}-b_{1}-2\right)\left(N-b_{0}\right)}\left[-2 O_{F}\left(b_{0}-1, b_{1}+2\right)\right. \\
\left.\quad \frac{2 \sqrt{2}}{\sqrt{3}} \frac{b_{1}+4}{b_{1}+2} O_{E}\left(b_{0}-1, b_{1}+2\right)-\frac{4 \sqrt{6}}{3} \sqrt{\frac{b_{1}+3}{b_{1}+1}} \frac{1}{b_{1}+2} O_{C}\left(b_{0}, b_{1}\right)\right]+\left(N-b_{0}-b_{1}-1\right) \times
\end{gathered}
$$




$$
\begin{gathered}
\times\left[\frac{4 \sqrt{6}}{3} \sqrt{\frac{b_{1}-1}{b_{1}+1}} \frac{1}{b_{1}} O_{C}\left(b_{0}+1, b_{1}-2\right)-\frac{2 \sqrt{6}}{3} \frac{b_{1}^{2}+b_{1}-2}{b_{1}\left(b_{1}+1\right)} O_{E}\left(b_{0}, b_{1}\right)+2 \frac{b_{1}-1}{b_{1}+1} O_{F}\left(b_{0}, b_{1}\right)\right] \\
+\left(N-b_{0}\right)\left[-\frac{2 \sqrt{6}}{3} \frac{\left(b_{1}+3\right) b_{1}}{\left(b_{1}+2\right)\left(b_{1}+1\right)} O_{E}\left(b_{0}, b_{1}\right)+2 \frac{\left(b_{1}+3\right)}{b_{1}+1} O_{F}\left(b_{0}, b_{1}\right)-\frac{4 \sqrt{6}}{3} \sqrt{\frac{b_{1}+3}{b_{1}+1}} \frac{1}{b_{1}+2} O_{H}\left(b_{0}-1, b_{1}+2\right)\right]
\end{gathered}
$$

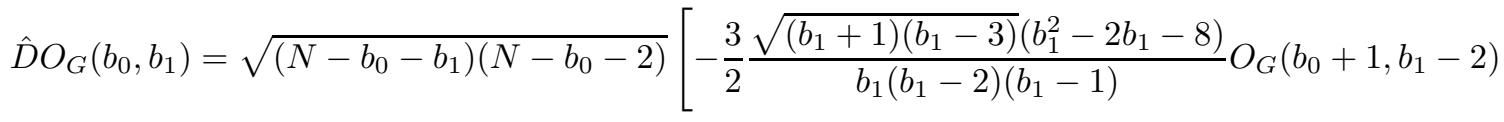

$$
\begin{aligned}
& \left.+\frac{\sqrt{3}}{2} \frac{\sqrt{\left(b_{1}+1\right)\left(b_{1}-3\right)}\left(b_{1}+2\right)}{\left(b_{1}-2\right)\left(b_{1}-1\right)} O_{H}\left(b_{0}+1, b_{1}-2\right)-6 \sqrt{\frac{b_{1}-3}{b_{1}-1}} \frac{\left(b_{1}+2\right)}{b_{1}\left(b_{1}-2\right)} O_{I}\left(b_{0}, b_{1}\right)\right] \\
& +\sqrt{\left(N-b_{0}-b_{1}-1\right)\left(N-b_{0}-1\right)}\left[18 \frac{\left(b_{1}^{2}-4\right)}{b_{1}^{2}\left(b_{1}^{2}-1\right)} O_{B}\left(b_{0}+1, b_{1}-2\right)\right. \\
& +2 \sqrt{3} \frac{\left(b_{1}^{2}-4\right)}{b_{1}^{2}\left(b_{1}^{2}-1\right)} O_{C}\left(b_{0}+1, b_{1}-2\right)-3 \sqrt{\frac{b_{1}-1}{b_{1}+1}} \frac{\left(b_{1}-2\right)\left(b_{1}+8\right)\left(b_{1}+3\right)}{b_{1}^{2}\left(b_{1}+2\right)\left(b_{1}-1\right)} O_{D}\left(b_{0}, b_{1}\right) \\
& +\sqrt{3} \sqrt{\frac{b_{1}-1}{b_{1}+1}} \frac{\left(b_{1}-2\right)\left(3 b_{1}+8\right)}{b_{1}^{2}\left(b_{1}+2\right)} O_{E}\left(b_{0}, b_{1}\right)-\frac{3}{2} \sqrt{\left(b_{1}+3\right)\left(b_{1}-1\right)} \frac{\left(b_{1}^{2}+2 b_{1}-8\right)}{b_{1}\left(b_{1}+1\right)\left(b_{1}+2\right)} O_{G}\left(b_{0}-1, b_{1}+2\right) \\
& \left.+\frac{\sqrt{3}}{2} \sqrt{\left(b_{1}+3\right)\left(b_{1}-1\right)} \frac{\left(b_{1}-2\right)}{\left(b_{1}+1\right)\left(b_{1}+2\right)} O_{H}\left(b_{0}-1, b_{1}+2\right)\right]+\left(N-b_{0}-b_{1}\right)\left[-\frac{\sqrt{3}}{2} \frac{b_{1}^{2}-b_{1}-6}{b_{1}\left(b_{1}-1\right)} O_{H}\left(b_{0}, b_{1}\right)\right. \\
& -\sqrt{3} \sqrt{\left(b_{1}+1\right)\left(b_{1}-1\right)} \frac{b_{1}^{2}-b_{1}-6}{\left(b_{1}-2\right)\left(b_{1}-1\right)^{2} b_{1}} O_{E}\left(b_{0}+1, b_{1}-2\right)+\frac{3}{2} \frac{\left(b_{1}-3\right)\left(b_{1}+2\right)^{2}}{b_{1}\left(b_{1}-2\right)\left(b_{1}-1\right)} O_{G}\left(b_{0}, b_{1}\right) \\
& \left.-3 \sqrt{\frac{b_{1}+1}{b_{1}-1}} \frac{b_{1}^{2}-b_{1}-6}{\left(b_{1}-2\right)\left(b_{1}-1\right) b_{1}} O_{D}\left(b_{0}+1, b_{1}-2\right)\right]+\left(N-b_{0}-1\right)\left[-\frac{\sqrt{3}}{2} \frac{\left(b_{1}-2\right)\left(b_{1}^{2}+b_{1}-8\right)}{b_{1}^{2}\left(b_{1}-1\right)} O_{H}\left(b_{0}, b_{1}\right)\right. \\
& +\frac{3}{2} \frac{\left(b_{1}-2\right)\left(b_{1}^{3}-b_{1}^{2}+6 b_{1}+48\right)}{b_{1}^{2}\left(b_{1}-1\right)\left(b_{1}+2\right)} O_{G}\left(b_{0}, b_{1}\right)+6 \sqrt{\frac{b_{1}-1}{b_{1}+1}} \frac{\left(b_{1}-2\right)\left(b_{1}-3\right)\left(b_{1}+2\right)}{b_{1}^{2}\left(b_{1}-1\right)^{2}} O_{D}\left(b_{0}+1, b_{1}-2\right) \\
& \left.-2 \sqrt{3} \sqrt{\left(b_{1}+1\right)\left(b_{1}-1\right)} \frac{b_{1}^{2}-4}{\left(b_{1}-1\right)^{2} b_{1}^{2}} O_{E}\left(b_{0}+1, b_{1}-2\right)+6 \sqrt{\frac{b_{1}+3}{b_{1}+1}} \frac{\left(b_{1}-2\right)}{b_{1}\left(b_{1}+2\right)} O_{I}\left(b_{0}-1, b_{1}+2\right)\right]
\end{aligned}
$$

$$
\begin{aligned}
\hat{D} O_{H}\left(b_{0}, b_{1}\right) & =\sqrt{\left(N-b_{0}-b_{1}\right)\left(N-b_{0}-2\right)}\left[\frac{\sqrt{3}}{2} \frac{\sqrt{\left(b_{1}+1\right)\left(b_{1}-3\right)}\left(b_{1}-4\right)}{\left(b_{1}-1\right) b_{1}} O_{G}\left(b_{0}+1, b_{1}-2\right)\right. \\
-\frac{1}{2} & \left.\frac{\sqrt{\left(b_{1}+1\right)\left(b_{1}-3\right)}}{b_{1}-1} O_{H}\left(b_{0}+1, b_{1}-2\right)+2 \sqrt{3} \frac{\sqrt{\left(b_{1}-1\right)\left(b_{1}-3\right)}}{b_{1}\left(b_{1}-1\right)} O_{I}\left(b_{0}, b_{1}\right)\right] \\
& +\sqrt{\left(N-b_{0}-b_{1}-1\right)\left(N-b_{0}-1\right)}\left[2 \sqrt{3} \frac{b_{1}^{2}-4}{b_{1}^{2}\left(b_{1}^{2}-1\right)} O_{B}\left(b_{0}+1, b_{1}-2\right)\right.
\end{aligned}
$$




$$
\begin{aligned}
& +2 \frac{3 b_{1}^{2}-4}{b_{1}^{2}\left(b_{1}^{2}-1\right)} O_{C}\left(b_{0}+1, b_{1}-2\right)-\frac{2}{\sqrt{3}} \sqrt{\frac{b_{1}-1}{b_{1}+1}} \frac{b_{1}^{3}+3 b_{1}^{2}-4 b_{1}-12}{\left(b_{1}^{2}-1\right) b_{1}^{2}} O_{D}\left(b_{0}, b_{1}\right) \\
& -2 \sqrt{\frac{b_{1}-1}{b_{1}+1}} \frac{\left(b_{1}+2\right)^{2}}{b_{1}^{2}\left(b_{1}+1\right)} O_{E}\left(b_{0}, b_{1}\right)+\frac{4 \sqrt{6}}{3} \sqrt{\frac{b_{1}-1}{b_{1}+1}} \frac{1}{b_{1}} O_{F}\left(b_{0}, b_{1}\right) \\
& +\sqrt{\frac{b_{1}-1}{b_{1}+1}} \frac{b_{1}+3}{b_{1}\left(b_{1}+1\right)}\left(-\sqrt{3} O_{D}\left(b_{0}, b_{1}\right)-O_{E}\left(b_{0}, b_{1}\right)\right) \\
& \left.+\frac{\sqrt{3}}{2} \sqrt{\left(b_{1}+3\right)\left(b_{1}-1\right)} \frac{b_{1}+4}{b_{1}\left(b_{1}+1\right)} O_{G}\left(b_{0}-1, b_{1}+2\right)-\frac{1}{2} \sqrt{\left(b_{1}+3\right)\left(b_{1}-1\right)} \frac{1}{b_{1}+1} O_{H}\left(b_{0}-1, b_{1}+2\right)\right] \\
& +\left(N-b_{0}-b_{1}\right)\left[\sqrt{3} \sqrt{\frac{b_{1}+1}{b_{1}-1}} \frac{\left(b_{1}-3\right)}{b_{1}\left(b_{1}-1\right)} O_{D}\left(b_{0}+1, b_{1}-2\right)+\sqrt{\frac{b_{1}+1}{b_{1}-1}} \frac{b_{1}-3}{b_{1}\left(b_{1}-1\right)} O_{E}\left(b_{0}+1, b_{1}-2\right)\right. \\
& \left.-\frac{\sqrt{3}}{2} \frac{b_{1}^{2}-b_{1}-6}{b_{1}\left(b_{1}-1\right)} O_{G}\left(b_{0}, b_{1}\right)+\frac{1}{2} \frac{b_{1}^{2}-5 b_{1}+6}{b_{1}\left(b_{1}-1\right)} O_{H}\left(b_{0}, b_{1}\right)\right]+\left(N-b_{0}-1\right) \times \\
& \times\left[\frac{2}{\sqrt{3}} \sqrt{\frac{b_{1}-1}{b_{1}+1}} \frac{b_{1}^{3}-3 b_{1}^{2}-4 b_{1}+12}{b_{1}^{2}\left(b_{1}-1\right)^{2}} O_{D}\left(b_{0}+1, b_{1}-2\right)+2 \frac{\left(b_{1}^{2}-4 b_{1}+4\right) \sqrt{\left(b_{1}+1\right)\left(b_{1}-1\right)}}{b_{1}^{2}\left(b_{1}-1\right)^{2}} O_{E}\left(b_{0}+1, b_{1}-2\right)\right. \\
& -\frac{4 \sqrt{6}}{3} \frac{\sqrt{\left(b_{1}+1\right)\left(b_{1}-1\right)}}{b_{1}\left(b_{1}-1\right)} O_{F}\left(b_{0}+1, b_{1}-2\right)-\frac{\sqrt{3}}{2} \frac{\left(b_{1}-2\right)\left(b_{1}^{2}+b_{1}-8\right)}{b_{1}^{2}\left(b_{1}-1\right)} O_{G}\left(b_{0}, b_{1}\right) \\
& \left.+\frac{b_{1}^{3}+3 b_{1}^{2}+10 b_{1}-16}{2 b_{1}^{2}\left(b_{1}-1\right)} O_{H}\left(b_{0}, b_{1}\right)-2 \sqrt{3} \sqrt{\frac{b_{1}+3}{b_{1}+1}} \frac{1}{b_{1}} O_{I}\left(b_{0}-1, b_{1}+2\right)\right] \\
& \hat{D} O_{I}\left(b_{0}, b_{1}\right)=\sqrt{\left(N-b_{0}-b_{1}\right)\left(N-b_{0}-2\right)}\left[12 \sqrt{\left(b_{1}+1\right)\left(b_{1}-3\right)} \frac{1}{b_{1}\left(b_{1}-2\right)\left(b_{1}-1\right)} O_{D}\left(b_{0}+1, b_{1}-2\right)\right. \\
& +4 \sqrt{3} \frac{\sqrt{\left(b_{1}+1\right)\left(b_{1}-3\right)}}{b_{1}\left(b_{1}-1\right)\left(b_{1}-2\right)} O_{E}\left(b_{0}+1, b_{1}-2\right)-6 \sqrt{\frac{b_{1}-3}{b_{1}-1}} \frac{b_{1}+2}{b_{1}\left(b_{1}-2\right)} O_{G}\left(b_{0}, b_{1}\right) \\
& \left.+2 \sqrt{3} \sqrt{\frac{b_{1}-3}{b_{1}-1}} \frac{1}{b_{1}} O_{H}\left(b_{0}, b_{1}\right)\right]+\left(N-b_{0}-2\right)\left[6 \frac{\sqrt{\left(b_{1}+1\right)\left(b_{1}-1\right)}\left(b_{1}-4\right)}{b_{1}\left(b_{1}-1\right)\left(b_{1}-2\right)} O_{G}\left(b_{0}+1, b_{1}-2\right)\right. \\
& \left.-2 \sqrt{3} \frac{\sqrt{\left(b_{1}+1\right)\left(b_{1}-1\right)}}{\left(b_{1}-2\right)\left(b_{1}-1\right)} O_{H}\left(b_{0}+1, b_{1}-2\right)+24 \frac{1}{b_{1}\left(b_{1}-2\right)} O_{I}\left(b_{0}, b_{1}\right)\right]
\end{aligned}
$$




\section{B. A Discussion on Intertwiners}

When $S^{n}$ acts on $V^{\otimes n} n>1$ it furnishes a reducible representation. Imagine that this includes the irreducible representations $R$ and $T$. Representing the action of $\sigma$ as a matrix $\Gamma(\sigma)$, in a suitable basis we can write

$$
\Gamma(\sigma)=\left[\begin{array}{ccc}
\Gamma_{R}(\sigma) & 0 & \cdots \\
0 & \Gamma_{S}(\sigma) & \cdots \\
\cdots & \cdots & \cdots
\end{array}\right]
$$

If we restrict ourselves to an $S_{n-1}$ subgroup of $S_{n}$, then in general, both $R$ and $S$ will subduce a number of representations. Assume for the sake of this discussion that $R$ subduces $R_{1}^{\prime}$ and $R_{2}^{\prime}$ and that $S$ subduces $S_{1}^{\prime}$ and $S_{2}^{\prime}$. Then, for $\sigma \in S_{n-1}$ we have

$$
\Gamma(\sigma)=\left[\begin{array}{ccccc}
\Gamma_{R_{1}^{\prime}}(\sigma) & 0 & 0 & 0 & \cdots \\
0 & \Gamma_{R_{2}^{\prime}}(\sigma) & 0 & 0 & \cdots \\
0 & 0 & \Gamma_{S_{1}^{\prime}}(\sigma) & 0 & \cdots \\
0 & 0 & 0 & \Gamma_{S_{2}^{\prime}}(\sigma) & \cdots \\
\ldots & \ldots & \ldots & \ldots & \cdots
\end{array}\right] .
$$

Imagine that $S_{1}^{\prime}=R_{1}^{\prime}$, that is, one of the irreducible representations subduced by $R$ is also subduced by $S$. Then, a simple application of the fundamental orthogonality relation gives

$$
\begin{aligned}
& \sum_{\sigma \in S_{n-1}}\left[\begin{array}{ccccc}
\Gamma_{R_{1}^{\prime}}(\sigma) & 0 & 0 & 0 & \cdots \\
0 & 0 & 0 & 0 & \cdots \\
0 & 0 & 0 & 0 & \cdots \\
0 & 0 & 0 & 0 & \cdots \\
\ldots & \ldots & \ldots & \ldots & \ldots
\end{array}\right]_{i j}\left[\begin{array}{ccccc}
0 & 0 & 0 & 0 & \cdots \\
0 & 0 & 0 & 0 & \cdots \\
0 & 0 & \Gamma_{S_{1}^{\prime}}(\sigma) & 0 & \cdots \\
0 & 0 & 0 & 0 & \cdots \\
\cdots & \ldots & \ldots & \ldots & \ldots
\end{array}\right]_{a b} \\
& =\frac{(n-1) !}{d_{R_{1}^{\prime}}} \delta_{R_{1}^{\prime} S_{1}^{\prime}}\left[\begin{array}{ccccc}
0 & 0 & \mathbf{1} & 0 & \cdots \\
0 & 0 & 0 & 0 & \cdots \\
0 & 0 & 0 & 0 & \ldots \\
0 & 0 & 0 & 0 & \ldots \\
\cdots & \ldots & \ldots & \ldots & \ldots
\end{array}\right]_{i b}\left[\begin{array}{ccccc}
0 & 0 & 0 & 0 & \cdots \\
0 & 0 & 0 & 0 & \cdots \\
\mathbf{1} & 0 & 0 & 0 & \ldots \\
0 & 0 & 0 & 0 & \ldots \\
\cdots & \ldots & \ldots & \ldots & \ldots
\end{array}\right]_{a j} \\
& \equiv \frac{(n-1) !}{d_{R_{1}^{\prime}}} \delta_{R_{1}^{\prime} S_{1}^{\prime}}\left(I_{R_{1}^{\prime} S_{1}^{\prime}}\right)_{i b}\left(I_{S_{1}^{\prime} R_{1}^{\prime}}\right)_{a j}
\end{aligned}
$$

where the form of the intertwiners has been spelled out. 


\section{References}

[1] S. Corley, A. Jevicki and S. Ramgoolam, "Exact correlators of giant gravitons from dual N = 4 SYM theory," Adv. Theor. Math. Phys. 5, 809 (2002) [arXiv:hep-th/0111222].

[2] V. Balasubramanian, D. Berenstein, B. Feng and M. x. Huang, "D-branes in Yang-Mills theory and emergent gauge symmetry," JHEP 0503, 006 (2005) [arXiv:hep-th/0411205].

[3] R. de Mello Koch, J. Smolic and M. Smolic, "Giant Gravitons - with Strings Attached (I)," JHEP 0706, 074 (2007), arXiv:hep-th/0701066.

[4] R. de Mello Koch, J. Smolic and M. Smolic, "Giant Gravitons - with Strings Attached (II)," JHEP 0709049 (2007), arXiv:hep-th/0701067.

[5] Y. Kimura and S. Ramgoolam, "Branes, Anti-Branes and Brauer Algebras in Gauge-Gravity duality," arXiv:0709.2158 [hep-th].

[6] D. Bekker, R. de Mello Koch and M. Stephanou, "Giant Gravitons - with Strings Attached (III)," arXiv:0710.5372 [hep-th].

[7] T. W. Brown, P. J. Heslop and S. Ramgoolam, "Diagonal multi-matrix correlators and BPS operators in N=4 SYM," arXiv:0711.0176 [hep-th].

[8] R. Bhattacharyya, S. Collins and R. d. M. Koch, "Exact Multi-Matrix Correlators," JHEP 0803, 044 (2008) [arXiv:0801.2061 [hep-th]].

[9] T. W. Brown, P. J. Heslop and S. Ramgoolam, "Diagonal free field matrix correlators, global symmetries and giant gravitons," arXiv:0806.1911 [hep-th].

[10] Y. Kimura and S. Ramgoolam, "Enhanced symmetries of gauge theory and resolving the spectrum of local operators," Phys. Rev. D 78, 126003 (2008) [arXiv:0807.3696 [hep-th]].

[11] Y. Kimura, "Non-holomorphic multi-matrix gauge invariant operators based on Brauer algebra," arXiv:0910.2170 [hep-th].

[12] S. Ramgoolam, "Schur-Weyl duality as an instrument of Gauge-String duality," AIP Conf. Proc. 1031, 255 (2008) [arXiv:0804.2764 [hep-th]].

[13] V. Balasubramanian, M. Berkooz, A. Naqvi and M. J. Strassler, "Giant gravitons in conformal field theory," JHEP 0204, 034 (2002) [arXiv:hep-th/0107119].

[14] G. 't Hooft, "A Planar Diagram Theory for Strong Interactions," Nucl. Phys. B 72, 461 (1974);

G. 't Hooft, "A Two-Dimensional Model For Mesons," Nucl. Phys. B 75, 461 (1974).

[15] R. de Mello Koch, "Geometries from Young Diagrams," JHEP 0811, 061 (2008) [arXiv:0806.0685 [hep-th]].

[16] R. de Mello Koch, N. Ives and M. Stephanou, "Correlators in Nontrivial Backgrounds," Phys. Rev. D 79, 026004 (2009) [arXiv:0810.4041 [hep-th]]. 
[17] R. de Mello Koch, T. K. Dey, N. Ives and M. Stephanou, "Correlators Of Operators with a Large R-charge," arXiv:0905.2273 [hep-th].

[18] H. Lin, O. Lunin and J. M. Maldacena, "Bubbling AdS space and 1/2 BPS geometries," JHEP 0410, 025 (2004) [arXiv:hep-th/0409174].

[19] V. Balasubramanian, J. de Boer, V. Jejjala and J. Simon, "The library of Babel: On the origin of gravitational thermodynamics," JHEP 0512, 006 (2005) [arXiv:hep-th/0508023], V. Balasubramanian, V. Jejjala and J. Simon, "The library of Babel," Int. J. Mod. Phys. D 14, 2181 (2005) [arXiv:hep-th/0505123].

[20] V. Balasubramanian, J. de Boer, V. Jejjala and J. Simon, "Entropy of near-extremal black holes in $\mathrm{AdS}_{5}$," JHEP 0805, 067 (2008) [arXiv:0707.3601 [hep-th]], R. Fareghbal, C. N. Gowdigere, A. E. Mosaffa and M. M. Sheikh-Jabbari, "Nearing Extremal Intersecting Giants and New Decoupled Sectors in N = 4 SYM," JHEP 0808, 070 (2008) [arXiv:0801.4457 [hep-th]].

[21] J. M. Maldacena, "The large N limit of superconformal field theories and supergravity," Adv. Theor. Math. Phys. 2, 231 (1998) [Int. J. Theor. Phys. 38, 1113 (1999)] [arXiv:hep-th/9711200];

S. S. Gubser, I. R. Klebanov and A. M. Polyakov, "Gauge theory correlators from non-critical string theory," Phys. Lett. B 428, 105 (1998) [arXiv:hep-th/9802109]; E. Witten, "Anti-de Sitter space and holography," Adv. Theor. Math. Phys. 2, 253 (1998) [arXiv:hep-th/9802150].

[22] K. Skenderis and M. Taylor, "Anatomy of bubbling solutions," JHEP 0709, 019 (2007) [arXiv:0706.0216 [hep-th]].

[23] D. E. Berenstein, J. M. Maldacena and H. S. Nastase, "Strings in flat space and pp waves from N = 4 super Yang Mills," JHEP 0204, 013 (2002) [arXiv:hep-th/0202021].

[24] H. Y. Chen, D. H. Correa and G. A. Silva, "Geometry and topology of bubble solutions from gauge theory," Phys. Rev. D 76, 026003 (2007) [arXiv:hep-th/0703068].

[25] R. de Mello Koch, T. K. Dey, N. Ives and M. Stephanou, "Hints of Integrability Beyond the Planar Limit," JHEP 1001, 014 (2010) [arXiv:0911.0967 [hep-th]].

[26] H. Lin, A. Morisse and J. P. Shock, "Strings on Bubbling Geometries," JHEP 1006, 055 (2010) [arXiv:1003.4190 [hep-th]].

[27] H. Lin, "Studies on 1/4 BPS and 1/8 BPS geometries," arXiv:1008.5307 [hep-th].

[28] J. McGreevy, L. Susskind and N. Toumbas, "Invasion of the giant gravitons from anti-de Sitter space," JHEP 0006, 008 (2000) [arXiv:hep-th/0003075];

M. T. Grisaru, R. C. Myers and O. Tafjord, "SUSY and Goliath," JHEP 0008, 040 (2000) [arXiv:hep-th/0008015];

A. Hashimoto, S. Hirano and N. Itzhaki, "Large branes in AdS and their field theory dual," JHEP 0008, 051 (2000) [arXiv:hep-th/0008016]. 
[29] D. Berenstein, "A toy model for the AdS/CFT correspondence," JHEP 0407, 018 (2004) [arXiv:hep-th/0403110].

[30] R. d. M. Koch, G. Mashile and N. Park, "Emergent Threebrane Lattices," Phys. Rev. D 81, 106009 (2010) [arXiv:1004.1108 [hep-th]].

[31] A. Mikhailov, "Giant gravitons from holomorphic surfaces," JHEP 0011, 027 (2000) [arXiv:hep-th/0010206].

[32] C. E. Beasley, "BPS branes from baryons," JHEP 0211, 015 (2002) [arXiv:hep-th/0207125].

[33] J. Kinney, J. M. Maldacena, S. Minwalla and S. Raju, "An index for 4 dimensional super conformal theories," Commun. Math. Phys. 275, 209 (2007) [arXiv:hep-th/0510251].

[34] I. Biswas, D. Gaiotto, S. Lahiri and S. Minwalla, "Supersymmetric states of N = 4 Yang-Mills from giant gravitons," JHEP 0712, 006 (2007) [arXiv:hep-th/0606087].

[35] G. Mandal and N. V. Suryanarayana, "Counting 1/8-BPS dual-giants," JHEP 0703, 031 (2007) [arXiv:hep-th/0606088].

[36] J. Pasukonis and S. Ramgoolam, "From counting to construction of BPS states in N=4 SYM," arXiv:1010.1683 [hep-th].

[37] V. Balasubramanian, M. x. Huang, T. S. Levi and A. Naqvi, "Open strings from N = 4 super Yang-Mills," JHEP 0208, 037 (2002) [arXiv:hep-th/0204196], O. Aharony, Y.E. Antebi, M. Berkooz and R. Fishman, "Holey sheets: Pfaffians and subdeterminants as D-brane operators in large $N$ gauge theories," JHEP 0212, 096 (2002) [arXiv:hep-th/0211152].

[38] D. Sadri and M. M. Sheikh-Jabbari, "Giant hedge-hogs: Spikes on giant gravitons," Nucl. Phys. B 687, 161 (2004) [arXiv:hep-th/0312155].

[39] D. Berenstein, "Shape and holography: Studies of dual operators to giant gravitons," Nucl. Phys. B 675, 179 (2003) [arXiv:hep-th/0306090].

[40] D. Berenstein, D. H. Correa and S. E. Vazquez, "A study of open strings ending on giant gravitons, spin chains and integrability," [arXiv:hep-th/0604123], D. Berenstein and S. E. Vazquez, "Integrable open spin chains from giant gravitons," JHEP 0506, 059 (2005) [arXiv:hep-th/0501078],

D. Berenstein, D. H. Correa and S. E. Vazquez, "Quantizing open spin chains with variable length: An example from giant gravitons," Phys. Rev. Lett. 95, 191601 (2005) [arXiv:hep-th/0502172],

D. H. Correa and G. A. Silva, "Dilatation operator and the Super Yang-Mills duals of open strings on AdS Giant Gravitons," [arXiv:hep-th/0608128].

[41] Y. Kimura, "Quarter BPS classified by Brauer algebra," JHEP 1005, 103 (2010) [arXiv:1002.2424 [hep-th]]. 
[42] E. D'Hoker and A. V. Ryzhov, "Three-point functions of quarter BPS operators in N = 4 SYM," JHEP 0202, 047 (2002) [arXiv:hep-th/0109065],

E. D'Hoker, P. Heslop, P. Howe and A. V. Ryzhov, "Systematics of quarter BPS operators in N = 4 SYM," JHEP 0304, 038 (2003) [arXiv:hep-th/0301104], P. J. Heslop and P. S. Howe, "OPEs and 3-point correlators of protected operators in N = 4 SYM," Nucl. Phys. B 626, 265 (2002) [arXiv:hep-th/0107212].

[43] T. W. Brown, "Permutations and the Loop," arXiv:0801.2094 [hep-th].

[44] T. W. Brown, "Cut-and-join operators and N=4 super Yang-Mills," arXiv:1002.2099 [hep-th],

[45] M. x. Huang, "Higher Genus BMN Correlators: Factorization and Recursion Relations," arXiv:1009.5447 [hep-th].

[46] N. Beisert, C. Kristjansen and M. Staudacher, "The dilatation operator of $N=4$ super Yang-Mills theory," Nucl. Phys. B 664, 131 (2003) [arXiv:hep-th/0303060].

[47] R. de Mello Koch and R. Gwyn, "Giant graviton correlators from dual SU(N) super Yang-Mills theory," JHEP 0411, 081 (2004) [arXiv:hep-th/0410236].

[48] R. Bhattacharyya, R. de Mello Koch and M. Stephanou, "Exact Multi-Restricted Schur Polynomial Correlators," arXiv:0805.3025 [hep-th].

[49] S. R. Das, A. Jevicki and S. D. Mathur, "Vibration modes of giant gravitons," Phys. Rev. D 63, 024013 (2001) [arXiv:hep-th/0009019].

[50] Andrei Okounkov and Anatoly Vershik, "A new approach to representation theory of symmetric groups," Selecta Mathematica, 2, 581-605, Andrei Okounkov and Anatoly Vershik, "A New Approach to the Representation Theory of the Symmetric Groups II," Journal of Mathematical Sciences 131, 5471-5494.

[51] R. C. Myers, "Dielectric-branes," JHEP 9912, 022 (1999) [arXiv:hep-th/9910053].

[52] W. Carlson, R. de Mello Koch and H. Lin, work in progress. 